\title{
Deformations compatibility equations in the general shell theory into the relative coordinate system with projected deformations
}

\author{
José Álvarez-Pérez ${ }^{1}$ and Milena Mesa-Lavista ${ }^{1 *}$ \\ 1 Universidad Autónoma de Nuevo León (UANL), School of Civil Engineering, Department of structural \\ engineering; San Nicolás de los Garza, México jose.alvarezpr@uanl.edu.mx; mmesal@uanl.edu.mx \\ * Correspondence: mmesal@uanl.edu.mx; Tel.: +52-8183-2940-00 ext.7240
}

\begin{abstract}
This paper presents a new analytical method for obtaining new deformations compatibility equations or, new Saint-Venant's identities, into the relative coordinate system with projected deformations by applying the hypothesis of the lineal shell theory in general flexion state. The method proposed generalizes the compatibility conditions established by A.L. Goldenveizer for the shell theory. On the other hand, the new equations include the deformations compatibility equations by other authors: Flügge, Saint-Venant, Love-Kirchhoff, Timoshenko, Goldenveizer, and Reissner-Mindlin. The results showed an increase of knowledge in general shell theory, and provide inverse and semi-inverse solutions, whose systems solution correspond to the hyper-statics degrees of the physical model, and not to their degrees of freedom.
\end{abstract}

Keywords: Saint-Venant's identities; compatibility equations; relative coordinate system; general shell theory.

\section{Introduction}

The "Relative coordinate method" (RCM) was developed for the calculus of shells (Figure 1) [1, 2] this method allows projecting the internal forces that appear in a shell with arbitrary geometry $\left(S^{*}\right)$ on top of another one with simpler geometry $(S)$ only if a scalar function (relative function) $f\left(\alpha_{1}, \alpha_{2}\right)$, that relates them as a continuous and bi-univocal form can be defined between them. The RCM, which is a generalization of Pücher's method, provides the possibility of using other reference surfaces with projected solicitations by selecting the most adequate reference surface or projected surface for the calculus of the same shell in each case [1-3].

The real surface of the Shell $\left(S^{*}\right)$ either in vectorial, Eq. (1), or parametric form, Eq. (2), is expressed according to the basic trihedron $\left(\overrightarrow{e_{1}}, \overrightarrow{e_{2}}, \overrightarrow{e_{N}}\right)$, the relative equation $f=f\left(\alpha_{1}, \alpha_{2}\right)$, the Lame's parameters $\left(A_{1}, A_{2}\right)$ and the relative relations between the two surfaces [1-3]. The fundamental expressions of the relative coordinate method are shown for parameters that characterize the reference surface $(S)$, Eq. (3),(1) and for the parameters that relate the two surfaces ( $S$ and $\left.S^{*}\right)$, Eq. (4). 


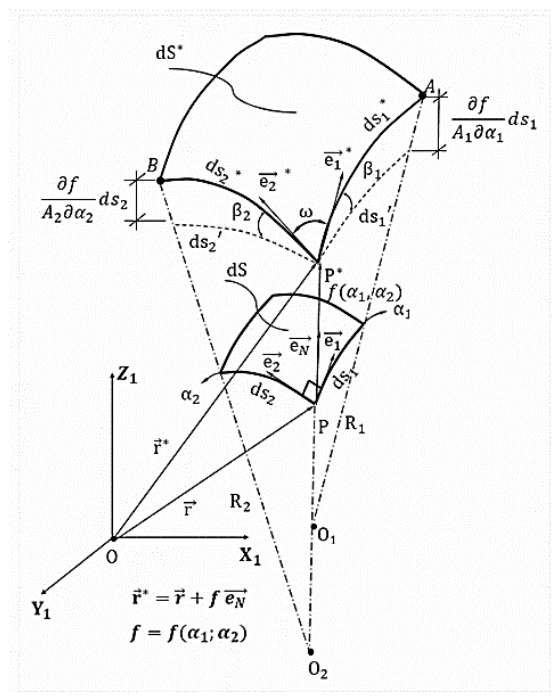

Figure 1. Relative coordinate between the middle real surface of the shell $\left(\boldsymbol{S}^{*}\right)$ and the reference surface or projected surface $(\boldsymbol{S})[1-3]$

$$
\begin{aligned}
& \overrightarrow{r^{*}}=\vec{r}\left(\alpha_{1}, \alpha_{2}\right)+f\left(\alpha_{1}, \alpha_{2}\right) \overrightarrow{e_{N}} \\
& S^{*}=\left\{\begin{array}{c}
X_{1}{ }^{*}=X_{1}\left(\alpha_{1}, \alpha_{2}\right) \\
Y_{1}{ }^{*}=Y_{1}\left(\alpha_{1}, \alpha_{2}\right) \\
Z_{1}{ }^{*}=Z_{1}\left(\alpha_{1}, \alpha_{2}\right)+f\left(\alpha_{1}, \alpha_{2}\right)
\end{array}\right. \\
& A_{1}=\left|\frac{\partial \vec{r}}{\partial \alpha_{1}}\right|, A_{2}=\left|\frac{\partial \vec{r}}{\partial \alpha_{2}}\right|, \overrightarrow{e_{1}}=\frac{1}{A_{1}} \frac{\partial \vec{r}}{\partial \alpha_{1}}, \overrightarrow{e_{2}}=\frac{1}{A_{2}} \frac{\partial \vec{r}}{\partial \alpha_{2}}, \overrightarrow{e_{N}}=\overrightarrow{e_{1}} \times \overrightarrow{e_{2}}=\frac{1}{A_{1} A_{2}} \frac{\partial \vec{r}}{\partial \alpha_{1}} \times \frac{\partial \vec{r}}{\partial \alpha_{2}}, \overrightarrow{e_{N}} \cdot \frac{\partial^{2} \vec{r}}{\partial \alpha_{1} \partial \alpha_{2}}=0 \\
& \rho_{1}=\frac{A_{1} A_{2}}{\frac{\partial A_{1}}{\partial \alpha_{2}}}, \rho_{2}=\frac{A_{1} A_{2}}{\frac{\partial A_{2}}{\partial \alpha_{1}}}, R_{1}=-\frac{A_{1}{ }^{2}}{\overrightarrow{e_{N}} \cdot \frac{\partial^{2} \vec{r}}{\partial \alpha_{1}{ }^{2}}}, R_{2}=-\frac{A_{2}{ }^{2}}{\overrightarrow{e_{N} \cdot \frac{\partial^{2} \vec{r}}{\partial \alpha_{2}}}} \\
& \left|d{\overrightarrow{r_{1}}}^{*}\right|=d s_{1}{ }^{*}=K_{1} A_{1} d \alpha_{1}=K_{1} d s_{1} ;\left|d{\overrightarrow{r_{2}}}^{*}\right|=d s_{2}{ }^{*}=K_{2} d s_{2}, \\
& K_{1}=\sqrt{\left[\left(1+\frac{f}{R_{1}}\right)^{2}+\left(\frac{\partial f}{A_{1} \partial \alpha_{1}}\right)^{2}\right]} ; \quad K_{2}=\sqrt{\left[\left(1+\frac{f}{R_{2}}\right)^{2}+\left(\frac{\partial f}{A_{2} \partial \alpha_{2}}\right)^{2}\right]} \\
& B_{1}=\tan \beta_{1}=\frac{\frac{1 \partial f}{A_{1} \partial \alpha_{1}}}{1+\frac{f}{R_{1}}} ; \quad B_{2}=\tan \beta_{2}=\frac{\frac{1}{A_{2} \partial f} \frac{\partial f}{2}}{1+\frac{f}{R_{2}}}, \\
& {\overrightarrow{e_{1}}}^{*}=\frac{d{\overrightarrow{r_{1}}}^{*}}{\left|d \overrightarrow{r_{1}}\right|}=\overrightarrow{e_{1}} \cos \beta_{1}+\overrightarrow{e_{N}} \sin \beta_{1} ;{\overrightarrow{e_{2}}}^{*}=\frac{d{\overrightarrow{r_{2}}}^{*}}{\left|d \overrightarrow{r_{2}}\right|}=\overrightarrow{e_{2}} \cos \beta_{2}+\overrightarrow{e_{N}} \sin \beta_{2} \\
& {\overrightarrow{e_{N}}}^{*}=\frac{\overrightarrow{e_{1}} x \overrightarrow{e_{2}}}{\sin w}=D\left(-B_{1} \overrightarrow{e_{1}}-B_{2} \overrightarrow{e_{2}}+\overrightarrow{e_{N}}\right) ; \quad D=\frac{\cos \beta_{1} \cos \beta_{2}}{\sin w}, d A^{*}=K_{1} K_{2} \sin w d A \\
& C_{11}=\frac{1}{R_{1}}+\frac{B_{1}^{2}}{R_{1}}-\frac{B_{2}}{\rho_{1}}-\frac{\partial B_{1}}{A_{1} \partial \alpha_{1}} ; \quad C_{22}=\frac{1}{R_{2}}+\frac{B_{2}^{2}}{R_{2}}-\frac{B_{1}}{\rho_{2}}-\frac{\partial B_{2}}{A_{2} \partial \alpha_{2}} ; C_{12}=\frac{B_{1} B_{2}}{R_{1}}+\frac{B_{1}}{\rho_{1}}-\frac{\partial B_{2}}{A_{1} \partial \alpha_{1}} ; \\
& C_{21}=\frac{B_{1} B_{2}}{R_{2}}+\frac{B_{2}}{\rho_{2}}-\frac{\partial B_{1}}{A_{2} \partial \alpha_{2}} ; \lambda_{1}=1+\frac{f}{R_{1}}=K_{1} \cos \beta_{1}, \lambda_{2}=1+\frac{f}{R_{2}}=K_{2} \cos \beta_{2}, \lambda_{2} C_{12}=\lambda_{1} C_{21}=C
\end{aligned}
$$

The value of the RCM depends on the generalization of the coordinate system in $\mathbb{R}^{3}[1,2,4]$ since it contains the following orthogonal coordinate systems: (a) Gauss's curvilinear intrinsic coordinate, imposing the condition $f=0$ on the relative relation, Eq. (3) and (4). (b) Cartesian coordinate, imposing the parametric condition of a Cartesian plane on the relative relation, Eq. (3) 
and (4). (c) Cylindrical-coordinate, imposing the parametric condition of a Polar plane on the relative relation, Eq. (3) and (4). (d) Spherical coordinate, imposing the parametrical condition of a Sphere on the relative relation, Eq. (3) and (4). (e) Cartesian intrinsic coordinate, imposing the parametrical condition of a coaxial cylinder on the relative relation, Eq. (3) and (4).

It can be said that when Goldenveizer's sufficient conditions $[5,6]$ are not fulfilled, then the membranal model (isostatic problem) plus the simple alteration effect does not provide satisfactory results in the internal equilibrium of the shell modeling $[4,6$, 7]. However, the state equations in the relative coordinate system with projected deformations that provide the mathematical operational models in displacements both for the Love-Kirchhoff's flexion (third order) and the Reissner-Mindlin (fifth order) were obtained [7].

Inverse and semi-inverse models make the solution system coincide with the hyper-statics degree of the physical model [4]. Since the solution systems are expressed in the relative coordinate system the reference surface that generates the most suitable analytical simplification in the shell mathematical modeling of arbitrary geometry $\left(S^{*}\right)$ can be obtained [4]. To get inverse and semiinverse mathematical models in the relative coordinate system with projected deformations, it is necessary to obtain not only Saint-Venant's compatibility equations of the deformations but also the stress functions. As it can be demonstrated [7] the conditions established by A. L. Goldenveizer [5, 6] are not sufficient for obtaining the deformations compatibility equations in the relative coordinate system, Eq. (5), as they are only established for Gauss's curvilinear orthogonal coordinate system [7].

The analytical method presented in this paper allows obtaining deformations compatibility equations or Saint-Venant's identities. This method generalizes the condition established by A. L. Goldenveizer, Eq. (5) for the shell lineal theory [5, 6].

$$
\begin{aligned}
& \frac{\partial}{\partial \alpha_{2}}\left(\frac{\partial \vec{U}}{\partial \alpha_{1}}\right)-\frac{\partial}{\partial \alpha_{1}}\left(\frac{\partial \vec{U}}{\partial \alpha_{2}}\right)=0 \\
& \frac{\partial}{\partial \alpha_{2}}\left(\frac{\partial \vec{\Omega}}{\partial \alpha_{1}}\right)-\frac{\partial}{\partial \alpha_{1}}\left(\frac{\partial \vec{\Omega}}{\partial \alpha_{2}}\right)=0
\end{aligned}
$$

\section{The new analytical method}

Twelve geometric equations, Eq.(6), in components of the shell's general flexion theory were obtained in relative coordinate system with projected deformations considering no dependency between the vectors $\vec{U}$ (displacement) and $\vec{\Omega}$ (rotations) [3, 7].

$$
\begin{gathered}
\text { (I) } \varepsilon_{1}=-\frac{B_{1}}{R_{1}} U_{1}+\frac{\partial U_{1}}{A_{1} \partial \alpha_{1}}+\frac{1}{\rho_{1}} U_{2}+\frac{1}{R_{1}} U_{N}+B_{1} \frac{\partial U_{N}}{A_{1} \partial \alpha_{1}} \\
\text { (II) } \varepsilon_{2}=\frac{1}{\rho_{2}} U_{1}-\frac{B_{2}}{R_{2}} U_{2}+\frac{\partial U_{2}}{A_{2} \partial \alpha_{2}}+\frac{1}{R_{2}} U_{N}+B_{2} \frac{\partial U_{N}}{A_{2} \partial \alpha_{2}} \\
\text { (III) } \gamma_{12}=-\left(\frac{1}{\rho_{1}}+\frac{B_{2}}{R_{1}}\right) U_{1}+\frac{\partial U_{2}}{A_{1} \partial \alpha_{1}}+B_{2} \frac{\partial U_{N}}{A_{1} \partial \alpha_{1}}+\psi_{2} B_{1} \lambda_{1}-\psi_{1} B_{2} \lambda_{1}+\delta \lambda_{1} \\
\text { (IV) } \gamma_{21}=\frac{\partial U_{1}}{A_{2} \partial \alpha_{2}}-U_{2}\left(\frac{1}{\rho_{2}}+\frac{B_{1}}{R_{2}}\right)+B_{1} \frac{\partial U_{N}}{A_{2} \partial \alpha_{2}}-\psi_{2} B_{1} \lambda_{2}+\psi_{1} B_{2} \lambda_{2}-\delta \lambda_{2} \\
\text { (V) } \gamma_{1 N}=-\frac{1}{R_{1}} U_{1}+\frac{\partial U_{N}}{A_{1} \partial \alpha_{1}}-\psi_{1} \lambda_{1} \\
\text { (VI) } \gamma_{2 N}=-\frac{1}{R_{2}} U_{2}+\frac{\partial U_{N}}{A_{2} \partial \alpha_{2}}-\psi_{2} \lambda_{2} \\
\text { (VII) } \varkappa_{1}=-\frac{\psi_{2}}{\rho_{1}}-\frac{\partial \psi_{1}}{A_{1} \partial \alpha_{1}} \\
\text { (VIII) } \varkappa_{2}=-\frac{\partial \psi_{2}}{A_{2} \partial \alpha_{2}}-\frac{\psi_{1}}{\rho_{2}}
\end{gathered}
$$




$$
\begin{gathered}
(I X) \varkappa_{12}=-\frac{\partial \psi_{2}}{A_{1} \partial \alpha_{1}}+\frac{\psi_{1}}{\rho_{1}}+\frac{\delta}{R_{1}} \\
(X) \varkappa_{21}=\frac{\psi_{2}}{\rho_{2}}-\frac{\partial \psi_{1}}{A_{2} \partial \alpha_{2}}-\frac{\delta}{R_{2}} \\
(X I) \tau_{1}=\frac{\psi_{2}}{R_{1}}+\frac{\partial \delta}{A_{1} \partial \alpha_{1}} \\
\text { (XII) } \tau_{2}=-\frac{\psi_{1}}{R_{2}}+\frac{\partial \delta}{A_{2} \partial \alpha_{2}}
\end{gathered}
$$

\subsection{Methodological procedure for the new analytical method}

The methodological steps for obtaining the deformations compatibility equations is as follows:

1. Defining the geometrical equations of the problem

2. Proposing an operator polynomial of degree $N\left(P_{i}{ }^{N}=P_{i}{ }^{N}\left(X_{1}, X_{2}, X_{3}, \ldots X_{\mathrm{m}}\right), \forall i, m \in \mathbb{N}\right)$, in the real variable as differential operator of each geometric equation

3. Operating each geometric equation according to the analytical structure of the operator polynomial previously proposed, until obtaining all $P^{(N)}{ }_{i}$, being $i$ the quantity of the geometric problem equations

4. Constructing the lineal combination between the operator polynomials $\left(P_{i}^{N}\right)$ until the fundamental identity is obtained $P^{(N)}{ }_{1}\left(\varepsilon_{1}\right)+P^{(N)}{ }_{2}\left(\varepsilon_{2}\right)+\cdots \sum_{i=1} P^{(N)}{ }_{i}\left(\varepsilon_{i}\right)=0, \forall i \in \mathbb{N}$

5. Applying the Indeterminate Coefficient Method (ICM).
a. Obtaining of the lineal homogenous equation system that provides the information of the fundamental identity in the preceding step
b. Resolution of the lineal homogenous equation system

6. Interpreting the solution of the lineal homogenous equation system

7. Obtaining of the deformations compatibility equations or Saint-Venant's identities

The new method (into the Euclidean vectorial space in $\mathbb{R}^{3}$ ) $[8,9]$ also permits obtaining the minimal degree of the operator polynomial that generates the sufficient conditions for a symmetric tensor of second order $E_{i j}$ (e.g. the tensor of Green-Lagrange) which corresponds to a deformation tensor and therefore can be integrated so that there is a displacement field from which it originates. This guarantees the continuity of the medium during the deformation process. Appendixes A, B, and $C$ show the application of the method for typical problems of the elasticity theory in two and threedimension Cartesian and Polar coordinate.

\subsection{Obtaining of the new deformations compatibility equation applying the new method}

1. Implementation of the geometric equations in relative coordinate with projected deformations, Eq. (6).

2. Proposition of the first-degree operator polynomial

$$
P_{i}^{(1)}=P^{(1)}\left(\alpha_{1}, \alpha_{2}\right)_{i}=\bar{C}_{i} \varepsilon_{i}+\bar{D}_{i} \frac{\partial \varepsilon_{i}}{\partial \alpha_{1}}+\bar{E}_{i} \frac{\partial \varepsilon_{i}}{\partial \alpha_{2}}, \forall i \in \mathbb{N}
$$

3. Derivation of each geometric equation of Eq. (6), according to the analytical structure of the operator polynomial proposed in Eq. (7), until obtaining all of $P^{(1)}{ }_{i}$. That is, the first geometric equation (I) of the Eq. (6): $\varepsilon_{1}=-\frac{B_{1}}{R_{1}} U_{1}+\frac{\partial U_{1}}{A_{1} \partial \alpha_{1}}+\frac{1}{\rho_{1}} U_{2}+\frac{1}{R_{1}} U_{N}+B_{1} \frac{\partial U_{N}}{A_{1} \partial \alpha_{1}}$ must be derivated successively, Eq. (8); obtaining the first operator polynomial $P^{(1)}{ }_{1}\left(\varepsilon_{1}\right)$, Eq. (9). Eq. (10) shows the other eleven, operator polynomials, of each geometric equation of Eq. (6).

$$
\begin{gathered}
\frac{\partial \varepsilon_{1}}{\partial \alpha_{1}}=-\frac{\partial}{\partial \alpha_{1}}\left(\frac{B_{1}}{R_{1}}\right) U_{1}+\left[-\frac{B_{1}}{R_{1}}+\frac{\partial}{\partial \alpha_{1}}\left(\frac{1}{A_{1}}\right)\right] \frac{\partial U_{1}}{\partial \alpha_{1}}+\frac{1}{A_{1}} \frac{\partial^{2} U_{1}}{\partial \alpha_{1}{ }^{2}}+\frac{\partial}{\partial \alpha_{1}}\left(\frac{1}{\rho_{1}}\right) U_{2}+\frac{1}{\rho_{1}} \frac{\partial U_{2}}{\partial \alpha_{1}}+\frac{\partial}{\partial \alpha_{1}}\left(\frac{1}{R_{1}}\right) U_{N}+ \\
{\left[\frac{1}{R_{1}}+\frac{\partial}{\partial \alpha_{1}}\left(\frac{B_{1}}{A_{1}}\right)\right] \frac{\partial U_{N}}{\partial \alpha_{1}}+\frac{B_{1}}{A_{1}} \frac{\partial^{2} U_{N}}{\partial \alpha_{1}{ }^{2}}}
\end{gathered}
$$




$$
\begin{gathered}
\frac{\partial \varepsilon_{1}}{\partial \alpha_{2}}=-\frac{\partial}{\partial \alpha_{2}}\left(\frac{B_{1}}{R_{1}}\right) U_{1}+\frac{\partial}{\partial \alpha_{2}}\left(\frac{1}{A_{1}}\right) \frac{\partial U_{1}}{\partial \alpha_{1}}-\frac{B_{1}}{R_{1}} \frac{\partial U_{1}}{\partial \alpha_{2}}+\frac{1}{A_{1}} \frac{\partial^{2} U_{1}}{\partial \alpha_{2} \partial \alpha_{1}}+\frac{\partial}{\partial \alpha_{2}}\left(\frac{1}{\rho_{1}}\right) U_{2}+\frac{1}{\rho_{1}} \frac{\partial U_{2}}{\partial \alpha_{2}}+\frac{\partial}{\partial \alpha_{2}}\left(\frac{1}{R_{1}}\right) U_{N}+ \\
\frac{\partial}{\partial \alpha_{2}}\left(\frac{B_{1}}{A_{1}}\right) \frac{\partial U_{N}}{\partial \alpha_{1}}+\frac{1}{R_{1}} \frac{\partial U_{N}}{\partial \alpha_{2}}+\frac{1}{R_{1}} \frac{\partial U_{N}}{\partial \alpha_{2}}+\frac{B_{1}}{A_{1}} \frac{\partial^{2} U_{N}}{\partial \alpha_{2} \partial \alpha_{1}} \\
P^{(1)}{ }_{1}\left(\varepsilon_{1}\right)=\bar{C}_{1} \varepsilon_{1}+\bar{D}_{1} \frac{\partial \varepsilon_{1}}{\partial \alpha_{1}}+\bar{E}_{1} \frac{\partial \varepsilon_{1}}{\partial \alpha_{2}}
\end{gathered}
$$

$P_{2}^{1}\left(\varepsilon_{2}\right), P_{3}^{1}\left(\gamma_{12}\right), P_{4}^{1}\left(\gamma_{21}\right), P_{5}^{1}\left(\gamma_{1 N}\right), P_{6}{ }^{1}\left(\gamma_{2 N}\right), P_{7}^{1}\left(\kappa_{1}\right), P_{8}^{1}\left(\kappa_{2}\right) P_{9}^{1}\left(\kappa_{12}\right), P_{10}{ }^{1}\left(\kappa_{21}\right) P_{11}{ }^{1}\left(\tau_{1}\right), P_{12}{ }^{1}\left(\tau_{2}\right)$

4. Creation of the lineal combination between the differential operators, Eq. (10), involved in the process until the formation of the fundamental identity, Eq. (11).

$$
\begin{gathered}
P_{1}^{1}\left(\varepsilon_{1}\right)+P_{2}{ }^{1}\left(\varepsilon_{2}\right)+P_{3}{ }^{1}\left(\gamma_{12}\right)+P_{4}{ }^{1}\left(\gamma_{21}\right)+P_{5}{ }^{1}\left(\gamma_{1 N}\right)+P_{6}{ }^{1}\left(\gamma_{2 N}\right)+ \\
P_{7}{ }^{1}\left(\kappa_{1}\right)+P_{8}{ }^{1}\left(\kappa_{2}\right)+P_{9}{ }^{1}\left(\kappa_{12}\right)+P_{10}{ }^{1}\left(\kappa_{21}\right)+P_{11}{ }^{1}\left(\tau_{1}\right)+P_{12}{ }^{1}\left(\tau_{2}\right)=0
\end{gathered}
$$

5. Applying the indeterminate coefficient method (ICM)

a. Obtaining of the lineal homogenous equation system that provides the formation of the fundamental identity in the previous step.

The resolution of the fundamental identity, Eq. (11), offered a linear homogenous equation system of sixty-six equations with seventy-two incognita, in which the difference provides six free variables indicating the number of deformations compatibility equations

b. Resolution of the lineal homogenous equation system

This resolution provided six free variables $\left(\overline{E_{3}}, \overline{D_{4}}, \overline{E_{5}}, \overline{E_{9}}, \overline{D_{10}}, \overline{D_{12}}\right)$ that constitute the fundamental solution system (F.S.S), Eq. (12). The eighteen coefficients obtained in Eq. (13) show the first-order differential ratios between the six geometric equations of Eq. (6)

$$
F . S . S=\left\{\left(\lambda \bar{E}_{3} ; \gamma \bar{D}_{4} ; \beta \bar{E}_{5} ; \phi \bar{E}_{9} ; \omega \bar{D}_{10} ; \kappa \bar{D}_{12}\right) ; \forall \lambda, \gamma, \beta, \phi, \omega, \kappa \in \mathbb{R}\right\}
$$

$$
\begin{gathered}
\left.11 \bar{E}_{11}=-\frac{A_{1}}{A_{2}} \bar{D}_{12} ; 2\right] \bar{E}_{7}=-\frac{A_{1}}{A_{2}} \bar{D}_{10} ; 3 \bar{D}_{8}=-\frac{A_{2}}{A_{1}} \bar{E}_{9} ; 4 \bar{E}_{1}=-\frac{A_{1}}{A_{2}} \bar{D}_{4} ; 5 \bar{D}_{2}=-\frac{A_{2}}{A_{1}} \bar{E}_{3} ; \\
6 \bar{D}_{6}=-\frac{A_{2}}{A_{1}} \bar{E}_{5} ; 7 \bar{C}_{11}=A_{1} \lambda_{2} \bar{D}_{4}+\frac{A_{1}}{R_{2}} \bar{D}_{10}-\frac{A_{1}}{\rho_{1}} \bar{D}_{12} ; \\
8 \bar{C}_{12}=-A_{2} \lambda_{1} \bar{E}_{3}-\frac{A_{2}}{R_{1}} \bar{E}_{9}-\frac{A_{2}}{\rho_{2}} \bar{E}_{11}
\end{gathered}
$$

$\rightarrow$ By substituting $\bar{E}_{11}$ of 1 in 8 we obtain: $\bar{C}_{12}=-A_{2} \lambda_{1} \bar{E}_{3}-\frac{A_{2}}{R_{1}} \bar{E}_{9}+\frac{A_{1}}{\rho_{2}} \bar{D}_{12}$

$$
9 \bar{C}_{8}=A_{2} B_{1} \lambda_{1} \bar{E}_{3}-\frac{A_{2}}{\rho_{2}} \bar{E}_{9}+\frac{A_{1}}{\rho_{1}} \bar{D}_{10}-\frac{A_{1}}{R_{1}} \bar{D}_{12} ; 10 \bar{C}_{7}=A_{1} B_{2} \lambda_{2} \bar{D}_{4}-\frac{A_{1}}{\rho_{1}} \bar{D}_{10}+\frac{A_{2}}{\rho_{2}} \bar{E}_{9}+\frac{A_{2}}{R_{2}} \bar{E}_{11}
$$

$\rightarrow$ By substituting $\bar{E}_{11}$ of 1 in 10 we obtain: $\bar{C}_{7}=A_{1} B_{2} \lambda_{2} \bar{D}_{4}-\frac{A_{1}}{\rho_{1}} \bar{D}_{10}+\frac{A_{2}}{\rho_{2}} \bar{E}_{9}-\frac{A_{1}}{R_{2}} \bar{D}_{12}$

$11 \bar{C}_{2}=\frac{A_{1}}{\rho_{1}} \bar{D}_{4}-\frac{A_{2}}{\rho_{2}} \bar{E}_{3} ; 12 \bar{C}_{1}=\frac{A_{2}}{\rho_{2}} \bar{E}_{3}-\frac{A_{1}}{\rho_{1}} \bar{D}_{4} ; 13 \bar{C}_{10}=-A_{2} B_{2} \lambda_{1} \bar{E}_{3}-A_{2} \lambda_{1} \bar{E}_{5}+\frac{A_{2}}{\rho_{1}} \bar{E}_{9}+\frac{A_{1}}{\rho_{2}} \bar{D}_{10} ;$

$$
14 \bar{C}_{9}=-A_{1} B_{1} \lambda_{2} \bar{D}_{4}-A_{1} \lambda_{2} \bar{D}_{6}+\frac{A_{1}}{\rho_{2}} \bar{D}_{10}+\frac{A_{2}}{\rho_{1}} \bar{E}_{9}
$$

$\rightarrow$ By substituting $\bar{D}_{6}$ of 6 in 14 we obtain: $\bar{C}_{9}=-A_{1} B_{1} \lambda_{2} \bar{D}_{4}+A_{2} \lambda_{2} \bar{E}_{5}+\frac{A_{1}}{\rho_{2}} \bar{D}_{10}+\frac{A_{2}}{\rho_{1}} \bar{E}_{9}$ 
$15 \overline{C_{4}}=A_{2}\left(\frac{1}{\rho_{1}}+\frac{B_{2}}{R_{1}}\right) \bar{E}_{3}-A_{1}\left(\frac{B_{1}}{R_{1}}-\frac{1}{\rho_{2}}\right) \bar{D}_{4}+\frac{A_{2}}{R_{1}} \bar{E}_{5} ; 16 \overline{C_{3}}=A_{1}\left(\frac{1}{\rho_{2}}+\frac{B_{1}}{R_{2}}\right) \bar{D}_{4}-A_{2}\left(\frac{B_{2}}{R_{2}}-\frac{1}{\rho_{1}}\right) \bar{E}_{3}+\frac{A_{1}}{R_{2}} \bar{D}_{6}$

$\rightarrow$ By substituting $\bar{D}_{6}$ of 6 in 16 we obtain: $\bar{C}_{3}=A_{1}\left(\frac{1}{\rho_{2}}+\frac{B_{1}}{R_{2}}\right) \bar{D}_{4}-A_{2}\left(\frac{B_{2}}{R_{2}}-\frac{1}{\rho_{1}}\right) \bar{E}_{3}-\frac{A_{2}}{R_{2}} \bar{E}_{5}$

$$
17 \bar{C}_{5}=A_{2} C_{22} \bar{E}_{3}-A_{1} C_{21} \bar{D}_{4}-\frac{A_{1} B_{2}}{R_{2}} \bar{D}_{6}+\frac{A_{2}}{\rho_{1}} \bar{E}_{5}
$$

$\rightarrow$ By substituting $\bar{D}_{6}$ of 6 in 17 we obtain: $\bar{C}_{5}=A_{2} C_{22} \bar{E}_{3}-A_{1} C_{21} \bar{D}_{4}+A_{2}\left(\frac{B_{2}}{R_{2}}+\frac{1}{\rho_{1}}\right) \bar{E}_{5}$

$$
18 \bar{C}_{6}=A_{1} C_{11} \bar{D}_{4}-A_{2} C_{12} \bar{E}_{3}-\frac{A_{2} B_{1}}{R_{1}} \bar{E}_{5}+\frac{A_{1}}{\rho_{2}} \bar{D}_{6}
$$

$\rightarrow$ By substituting $\bar{D}_{6}$ of 6 in 18 we obtain: $\bar{C}_{6}=A_{1} C_{11} \bar{D}_{4}-A_{2} C_{12} \bar{E}_{3}-A_{2}\left(\frac{B_{1}}{R_{1}}+\frac{1}{\rho_{2}}\right) \bar{E}_{5}$

6. Interpreting the lineal homogenous equation system

The six free variables $\left(\overline{E_{3}}, \overline{D_{4}}, \overline{E_{5}}, \overline{E_{9}}, \overline{D_{10}}, \overline{D_{12}}\right)$ obtained in the resolution of the lineal homogenous equation system provide the six deformations compatibility equations into the relative coordinate system with projected deformations. To obtain each compatibility equation of the deformations independently one from the other, it is necessary the contribution of every single free variable by annulling the others. For instance, to obtain the contribution of the free variable $\bar{E}_{3}$, the other variables are annulled, that is: $\bar{D}_{4}=\bar{E}_{5}=\bar{D}_{10}=\bar{D}_{12}=0$. By substituting $\bar{D}_{4}=\bar{E}_{5}=\bar{D}_{10}=$ $\bar{D}_{12}=0$ in the general relations, Eq. (13), the coefficients related to the free variable $\bar{E}_{3}$ are obtained, Eq. (14).

$$
\begin{aligned}
& 11 \bar{C}_{1}=\frac{A_{2}}{\rho_{2}} \bar{E}_{3}, 2 \bar{C}_{2}=-\frac{A_{2}}{\rho_{2}}, 3 \bar{C}_{3}=-A_{2}\left(\frac{B_{2}}{R_{2}}-\frac{1}{\rho_{1}}\right), 4 \bar{C}_{4}=A_{2}\left(\frac{1}{\rho_{1}}+\frac{B_{2}}{R_{1}}\right) \bar{E}_{3}, 5 \bar{C}_{5}=A_{2} C_{22} \bar{E}_{3} \\
& 6 \bar{C}_{6}=-A_{2} C_{12} \bar{E}_{3}, 7 \bar{C}_{8}=A_{2} B_{1} \lambda_{1} \bar{E}_{3}, 8 \bar{C}_{10}=-A_{2} B_{2} \lambda_{1} \bar{E}_{3}, 9 \bar{C}_{12}=-A_{2} \lambda_{1} \bar{E}_{3}, 10 \bar{D}_{2}=-\frac{A_{2}}{A_{1}} \bar{E}_{3}
\end{aligned}
$$

By orderly adding the coefficients obtained, Eq. (14) and taking into account the analytical structure of the operator polynomial, Eq. (7), is gets the first deformations compatibility equation, Eq. (15):

$$
\begin{aligned}
& \underbrace{\frac{A_{2}}{\rho_{2}}}_{\tilde{C}_{1}} \varepsilon_{1}-\underbrace{\frac{A_{2}}{\rho_{2}}}_{\tilde{C}_{2}} \varepsilon_{2} \underbrace{-A_{2}\left(\frac{B_{2}}{R_{2}}-\frac{1}{\rho_{1}}\right)}_{\widetilde{C_{3}}} \gamma_{12}+\underbrace{+A_{2}\left(\frac{1}{\rho_{1}}+\frac{B_{2}}{R_{1}}\right)}_{\widetilde{C}_{4}} \gamma_{21}+\underbrace{A_{2} C_{22}}_{\widetilde{C_{5}}} \gamma_{1 N}-\underbrace{-A_{2} C_{12}}_{\widetilde{C_{6}}} \gamma_{2 N}+\underbrace{+A_{2} B_{1} \lambda_{1}}_{\widetilde{C_{8}}} \kappa_{2} \\
& \underbrace{-A_{2} B_{2} \lambda_{1}}_{\bar{C}_{10}} \kappa_{21}-\underbrace{A_{2} \lambda_{1}}_{\bar{C}_{12}} \tau_{2}-\underbrace{-\frac{A_{2}}{A_{2}}}_{\bar{D}_{2}} \frac{\partial \varepsilon_{2}}{\partial \alpha_{1}}=0
\end{aligned}
$$

Where: $\overline{\mathrm{C}}_{\mathrm{i}}$ : Coefficient that accompanies the geometric equation $i$; $\overline{\mathrm{D}}_{\mathrm{i}}$ : Coefficient that accompanies the partial derivative with respect to coordinate $\alpha_{1} ; \overline{\mathrm{E}}_{\mathrm{i}}$ : Coefficient that accompanies the partial derivative with respect to coordinate $\alpha_{2}$.

If Eq. (15) is operate the by $\frac{1}{\mathrm{~A}_{2}}$ will obtain Eq. (16):

$$
\begin{gathered}
\frac{1}{\rho_{2}} \varepsilon_{1}-\frac{1}{\rho_{2}} \varepsilon_{2}-\frac{1}{A_{1}} \frac{\partial \varepsilon_{2}}{\partial \alpha_{1}}-\left(\frac{B_{2}}{R_{2}}-\frac{1}{\rho_{1}}\right) \gamma_{12}+\frac{1}{A_{2}} \frac{\partial \gamma_{12}}{\partial \alpha_{2}}+\left(\frac{1}{\rho_{1}}+\frac{B_{2}}{R_{1}}\right) \gamma_{21}+C_{22} \gamma_{1 N}-C_{12} \gamma_{2 N}+B_{1} \lambda_{1} \kappa_{2}-B_{2} \lambda_{1} \kappa_{21}- \\
\lambda_{1} \tau_{2}=0
\end{gathered}
$$

The other five deformations compatibility equations are similarly obtained, Eqs. (17)-(21).

The free variable $\overline{\mathrm{D}}_{4}$ provides the second equation, Eq. (17):

$$
\begin{gathered}
\frac{1}{\rho_{1}} \varepsilon_{1}+\frac{1}{A_{2}} \frac{\partial \varepsilon_{1}}{\partial \alpha_{2}}-\frac{1}{\rho_{1}} \varepsilon_{2}-\left(\frac{1}{\rho_{2}}+\frac{B_{1}}{R_{2}}\right) \gamma_{12}+\left(\frac{B_{1}}{R_{1}}-\frac{1}{\rho_{2}}\right) \gamma_{21}-\frac{1}{A_{1}} \frac{\partial \gamma_{21}}{\partial \alpha_{1}}+C_{21} \gamma_{1 N}-C_{11} \gamma_{2 N}-B_{2} \lambda_{2} \kappa_{1}+B_{1} \lambda_{2} \kappa_{12} \\
-\lambda_{2} \tau_{1}=0
\end{gathered}
$$

The free variable $\overline{\mathrm{E}}_{5}$ provides the third equation, Eq. (18): 


$$
\frac{1}{R_{2}} \gamma_{12}-\frac{1}{R_{1}} \gamma_{21}-\left(\frac{B_{2}}{R_{2}}+\frac{1}{\rho_{1}}\right) \gamma_{1 N}-\frac{1}{A_{2}} \frac{\partial \gamma_{1 N}}{\partial \alpha_{2}}+\left(\frac{B_{1}}{R_{1}}+\frac{1}{\rho_{2}}\right) \gamma_{2 N}+\frac{1}{A_{1}} \frac{\partial \gamma_{2 N}}{\partial \alpha_{1}}-\lambda_{2} \kappa_{12}+\lambda_{1} \kappa_{21}=0
$$

The free variable $\overline{\mathrm{E}}_{9}$ provides the fourth equation, Eq. (19):

$$
\frac{1}{\rho_{2}} \kappa_{1}-\frac{1}{\rho_{2}} \kappa_{2}-\frac{1}{A_{1}} \frac{\partial \kappa_{2}}{\partial \alpha_{1}}+\frac{1}{\rho_{1}} \kappa_{12}+\frac{1}{A_{2}} \frac{\partial \kappa_{12}}{\partial \alpha_{2}}+\frac{1}{\rho_{1}} \kappa_{21}-\frac{1}{R_{1}} \tau_{2}=0
$$

The free variable $\overline{\mathrm{D}}_{10}$ provides the fifth equation, Eq. (20):

$$
\frac{1}{\rho_{1}} \kappa_{1}+\frac{1}{A_{2}} \frac{\partial \kappa_{1}}{\partial \alpha_{2}}-\frac{1}{\rho_{1}} \kappa_{2}-\frac{1}{\rho_{2}} \kappa_{12}-\frac{1}{\rho_{2}} \kappa_{21}-\frac{1}{A_{1}} \frac{\partial \kappa_{21}}{\partial \alpha_{1}}-\frac{1}{R_{2}} \tau_{1}=0
$$

The free variable $\overline{\mathrm{D}}_{12}$ provides the sixth equation, Eq. (21):

$$
\frac{1}{R_{2}} \kappa_{1}+\frac{1}{R_{1}} \kappa_{2}+\frac{1}{\rho_{1}} \tau_{1}+\frac{1}{A_{2}} \frac{\partial \tau_{1}}{\partial \alpha_{2}}-\frac{1}{\rho_{2}} \tau_{2}-\frac{1}{A_{1}} \frac{\partial \tau_{2}}{\partial \alpha_{1}}=0
$$

\section{Results and discussion: Particular cases and theoretical results}

The new general deformations compatibility equations were obtained by applying the new method in the general shell flexion theory into the relative coordinate system with projected deformations, Eq. (22).

$$
\begin{aligned}
& \langle 1\rangle \frac{1}{\rho_{2}} \varepsilon_{1}-\frac{1}{\rho_{2}} \varepsilon_{2}-\frac{1}{A_{1}} \frac{\partial \varepsilon_{2}}{\partial \alpha_{1}}-\left(\frac{B_{2}}{R_{2}}-\frac{1}{\rho_{1}}\right) \gamma_{12}+\frac{1}{A_{2}} \frac{\partial \gamma_{12}}{\partial \alpha_{2}}+\left(\frac{1}{\rho_{1}}+\frac{B_{2}}{R_{1}}\right) \gamma_{21}+C_{22} \gamma_{1 N}-C_{12} \gamma_{2 N}+B_{1} \lambda_{1} \kappa_{2}-B_{2} \lambda_{1} \kappa_{21}- \\
& \lambda_{1} \tau_{2}=0 \\
& \langle 2\rangle \frac{1}{\rho_{1}} \varepsilon_{1}+\frac{1}{A_{2}} \frac{\partial \varepsilon_{1}}{\partial \alpha_{2}}-\frac{1}{\rho_{1}} \varepsilon_{2}-\left(\frac{1}{\rho_{2}}+\frac{B_{1}}{R_{2}}\right) \gamma_{12}+\left(\frac{B_{1}}{R_{1}}-\frac{1}{\rho_{2}}\right) \gamma_{21}-\frac{1}{A_{1}} \frac{\partial \gamma_{21}}{\partial \alpha_{1}}+C_{21} \gamma_{1 N}-C_{11} \gamma_{2 N}-B_{2} \lambda_{2} \kappa_{1} \\
& +B_{1} \lambda_{2} \kappa_{12}-\lambda_{2} \tau_{1}=0 \\
& \langle 3\rangle \frac{1}{R_{2}} \gamma_{12}-\frac{1}{R_{1}} \gamma_{21}-\left(\frac{B_{2}}{R_{2}}+\frac{1}{\rho_{1}}\right) \gamma_{1 N}-\frac{1}{A_{2}} \frac{\partial \gamma_{1 N}}{\partial \alpha_{2}}+\left(\frac{B_{1}}{R_{1}}+\frac{1}{\rho_{2}}\right) \gamma_{2 N}+\frac{1}{A_{1}} \frac{\partial \gamma_{2 N}}{\partial \alpha_{1}}-\lambda_{2} \kappa_{12}+\lambda_{1} \kappa_{21}=0 \\
& \langle 4\rangle \frac{1}{\rho_{2}} \kappa_{1}-\frac{1}{\rho_{2}} \kappa_{2}-\frac{1}{A_{1}} \frac{\partial \kappa_{2}}{\partial \alpha_{1}}+\frac{1}{\rho_{1}} \kappa_{12}+\frac{1}{A_{2}} \frac{\partial \kappa_{12}}{\partial \alpha_{2}}+\frac{1}{\rho_{1}} \kappa_{21}-\frac{\tau_{2}}{R_{1}}=0 \\
& \langle 5\rangle \frac{1}{\rho_{1}} \kappa_{1}+\frac{1}{A_{2}} \frac{\partial \kappa_{1}}{\partial \alpha_{2}}-\frac{1}{\rho_{1}} \kappa_{2}-\frac{1}{\rho_{2}} \kappa_{12}-\frac{1}{\rho_{2}} \kappa_{21}-\frac{1}{A_{1}} \frac{\partial \kappa_{21}}{\partial \alpha_{1}}-\frac{\tau_{1}}{R_{2}}=0 \\
& \langle 6\rangle \frac{1}{R_{2}} \kappa_{1}+\frac{1}{R_{1}} \kappa_{2}+\frac{1}{\rho_{1}} \tau_{1}+\frac{1}{A_{2}} \frac{\partial \tau_{1}}{\partial \alpha_{2}}-\frac{1}{\rho_{2}} \tau_{2}-\frac{1}{A_{1}} \frac{\partial \tau_{2}}{\partial \alpha_{1}}=0
\end{aligned}
$$

The proposed method has a cyclic character, as each proposal of the operator polynomial of degree $N\left(P_{i}{ }^{N}\right)$ represents one solution, either trivial (null) or not. One of the purposes of this method is to find the minimal degree of the operator polynomial that generates the nontrivial solution. 


\subsection{Reissner-Mindlin's deformations compatibility equations in Gauss's intrinsic coordinates}

By substituting the condition $f=0$ in the new general deformations compatibility equations, Eq. (22), Reissner-Mindlin's deformations compatibility equations of the thick shell theory are obtained, Eq.(23) [4, 10-12]. The condition $f=0$ indicates that the reference surface in Gauss's intrinsic coordinates matches with the middle shell surface $\left(S^{*}\right)$ (Figure 1), Eq. (24).

$$
\begin{gathered}
\text { (1) } \frac{1}{\rho_{2}} \varepsilon_{1}-\frac{1}{\rho_{2}} \varepsilon_{2}-\frac{1}{A_{1}} \frac{\partial \varepsilon_{2}}{\partial \alpha_{1}}+\frac{\gamma_{12}}{\rho_{1}}+\frac{1}{A_{2}} \frac{\partial \gamma_{12}}{\partial \alpha_{2}}+\frac{\gamma_{21}}{\rho_{1}}+\frac{\gamma_{1 N}}{R_{2}}-\tau_{2}=0 \\
\text { (2) } \frac{1}{\rho_{1}} \varepsilon_{1}+\frac{1}{A_{2}} \frac{\partial \varepsilon_{1}}{\partial \alpha_{2}}-\frac{1}{\rho_{1}} \varepsilon_{2}-\frac{\gamma_{12}}{\rho_{2}}-\frac{\gamma_{21}}{\rho_{2}}-\frac{1}{A_{1}} \frac{\partial \gamma_{21}}{\partial \alpha_{1}}-\frac{\gamma_{2 N}}{R_{1}}-\tau_{1}=0 \\
\text { (3) } \frac{\gamma_{12}}{R_{2}}-\frac{\gamma_{21}}{R_{1}}-\frac{\gamma_{1 N}}{\rho_{1}}-\frac{1}{A_{2}} \frac{\partial \gamma_{1 N}}{\partial \alpha_{2}}+\frac{\gamma_{2 N}}{\rho_{2}}+\frac{1}{A_{1}} \frac{\partial \gamma_{2 N}}{\partial \alpha_{1}}-\kappa_{12}+\kappa_{21}=0 \\
\text { (4) } \frac{1}{\rho_{2}} \kappa_{1}-\frac{1}{\rho_{2}} \kappa_{2}-\frac{1}{A_{1}} \frac{\partial \kappa_{2}}{\partial \alpha_{1}}+\frac{1}{\rho_{1}} \kappa_{12}+\frac{1}{A_{2}} \frac{\partial \kappa_{12}}{\partial \alpha_{2}}+\frac{1}{\rho_{1}} \kappa_{21}-\frac{\tau_{2}}{R_{1}}=0 \\
\text { (5) } \frac{1}{\rho_{1}} \kappa_{1}+\frac{1}{A_{2}} \frac{\partial \kappa_{1}}{\partial \alpha_{2}}-\frac{1}{\rho_{1}} \kappa_{2}-\frac{1}{\rho_{2}} \kappa_{12}-\frac{1}{\rho_{2}} \kappa_{21}-\frac{1}{A_{1}} \frac{\partial \kappa_{21}}{\partial \alpha_{1}}-\frac{\tau_{1}}{R_{2}}=0 \\
\text { (6) } \frac{1}{R_{2}} \kappa_{1}+\frac{1}{R_{1}} \kappa_{2}+\frac{1}{\rho_{1}} \tau_{1}+\frac{1}{A_{2}} \frac{\partial \tau_{1}}{\partial \alpha_{2}}-\frac{1}{\rho_{2}} \tau_{2}-\frac{1}{A_{1}} \frac{\partial \tau_{2}}{\partial \alpha_{1}}=0 \\
f=0 \Rightarrow B_{1}=B_{2}=\beta_{1}=\beta_{2}=0, \quad K_{1}=K_{2}=1 \Rightarrow \lambda_{1}=\left(1+\frac{f}{R_{1}}\right)=K_{1} \cos \beta_{1}=1, \\
\lambda_{2}=\left(1+\frac{f}{R_{2}}\right)=K_{2} \cos \beta_{2}=1
\end{gathered}
$$

\subsection{Love-Kirchhoff's deformations compatibility equations in Gauss's intrinsic coordinate}

By substituting the thin shell condition $\gamma_{1 \mathrm{~N}}=\gamma_{2 \mathrm{~N}}=0$ in Eq. (23) we obtain Love-Kirchhoff's compatibility equations of the deformations that belong to the thin shell theory, Eq. (25) [5, 6, 10-15]. The most recent researches on the flexion theory of cylindrical thin shells is shown in Eq. (25) for its geometric description [28].

$$
\begin{aligned}
& \text { (1) } \frac{1}{\rho_{2}} \varepsilon_{1}-\frac{1}{\rho_{2}} \varepsilon_{2}-\frac{1}{A_{1}} \frac{\partial \varepsilon_{2}}{\partial \alpha_{1}}+\frac{1}{\rho_{1}} \gamma_{12}+\frac{1}{A_{2}} \frac{\partial \gamma_{12}}{\partial \alpha_{2}}+\frac{1}{\rho_{1}} \gamma_{21}-\tau_{2}=0 \\
& \text { (2) } \frac{1}{\rho_{1}} \varepsilon_{1}+\frac{1}{A_{2}} \frac{\partial \varepsilon_{1}}{\partial \alpha_{2}}-\frac{1}{\rho_{1}} \varepsilon_{2}-\frac{1}{\rho_{2}} \gamma_{12}-\frac{1}{\rho_{2}} \gamma_{21}-\frac{1}{A_{1}} \frac{\partial \gamma_{21}}{\partial \alpha_{1}}-\tau_{1}=0 \\
& \text { (3) } \frac{1}{R_{2}} \gamma_{12}-\frac{1}{R_{1}} \gamma_{21}-\kappa_{12}+\kappa_{21}=0 \\
& \text { (4) } \frac{1}{\rho_{2}} \kappa_{1}-\frac{1}{\rho_{2}} \kappa_{2}-\frac{1}{A_{1}} \frac{\partial \kappa_{2}}{\partial \alpha_{1}}+\frac{1}{\rho_{1}} \kappa_{12}+\frac{1}{A_{2}} \frac{\partial \kappa_{12}}{\partial \alpha_{2}}+\frac{1}{\rho_{1}} \kappa_{21}-\frac{\tau_{2}}{R_{1}}=0 \\
& \text { (5) } \frac{1}{\rho_{1}} \kappa_{1}+\frac{1}{A_{2}} \frac{\partial \kappa_{1}}{\partial \alpha_{2}}-\frac{1}{\rho_{1}} \kappa_{2}-\frac{1}{\rho_{2}} \kappa_{12}-\frac{1}{\rho_{2}} \kappa_{21}-\frac{1}{A_{1}} \frac{\partial \kappa_{21}}{\partial \alpha_{1}}-\frac{\tau_{1}}{R_{2}}=0 \\
& \text { (6) } \frac{1}{R_{2}} \kappa_{1}+\frac{1}{R_{1}} \kappa_{2}+\frac{1}{\rho_{1}} \tau_{1}+\frac{1}{A_{2}} \frac{\partial \tau_{1}}{\partial \alpha_{2}}-\frac{1}{\rho_{2}} \tau_{2}-\frac{1}{A_{1}} \frac{\partial \tau_{2}}{\partial \alpha_{1}}=0
\end{aligned}
$$

The equations from Eq. (25) correspond to the equations 20.11 obtained by Goldenveizer [5, 6 , 10] when the shell twist curvature $\kappa_{12}$ is disregarded being $\gamma_{12}=w^{(1)}, \gamma_{21}=-w^{(2)}$ the angular deformations assumed by Goldenveizer [5,6]. Overlooking the shear deformations $\gamma_{1 N}$ and $\gamma_{2 N}$ in Love-Kirchhoff's theory of the thin shell implies a dependence ratio between the lineal displacement vectors $(\vec{U})$ and the rotations $(\vec{\Omega})$, Eq. (26) [7]. 


$$
\left.\begin{array}{rl}
\Psi_{1} & =\frac{\partial U_{N}}{A_{1} \partial \alpha_{1}}-\frac{1}{R_{1}} U_{1} \\
\Psi_{2} & =\frac{\partial U_{N}}{A_{2} \partial \alpha_{2}}-\frac{1}{R_{2}} U_{2} \\
\left.\mathrm{w}_{1}\right) & =\frac{1}{2 A_{1} A_{2}}\left(-\frac{\partial\left(A_{2} U_{2}\right)}{\partial \alpha_{1}}+\frac{\partial\left(A_{1} U_{1}\right)}{\partial \alpha_{2}}\right)
\end{array}\right\} \Rightarrow \begin{gathered}
\text { Relation between } \vec{U} \text { and } \vec{\Omega} \\
\vec{\Omega}=\frac{1}{2} \vec{\nabla} \times \vec{U}
\end{gathered}
$$

\subsection{New compatibility equations of the projected deformations in Cartesian coordinate system}

New compatibility equations of the projected deformations on a Cartesian plane, were obtained, Eq. (28), by substituting the geometric characteristics, Eq. (27), in the new general compatibility equations of the deformations, Eq. (22), (Figure 2). In Eq. (28), the six compatibility equations satisfy themselves identically. These equations may be used for the construction of the second-order new surface with inverse formulations (parabola, ellipse, etc.) [11-15].

$$
\begin{gathered}
\alpha_{1}=x ; \alpha_{2}=y ; f=z(x, y) ; A_{1}=A_{2}=\lambda_{1}=\lambda_{2}=1 ; R_{1}=R_{2}=\rho_{1}=\rho_{2}=\infty \\
B_{1}=\frac{\partial z}{\partial x} ; B_{2}=\frac{\partial z}{\partial y} ; C_{11}=-\frac{\partial^{2} z}{\partial x^{2}} ; C_{22}=-\frac{\partial^{2} z}{\partial y^{2}} ; C_{12}=C_{21}=-\frac{\partial^{2} z}{\partial x \partial y} \\
\text { (1) } \frac{\partial \varepsilon_{y}}{\partial x}+\frac{\partial \gamma_{x y}}{\partial y}-\frac{\partial^{2} z}{\partial y^{2}} \gamma_{x N}+\frac{\partial^{2} z}{\partial x \partial y} \gamma_{y N}+\frac{\partial z}{\partial x} \kappa_{y}-\frac{\partial z}{\partial y} \kappa_{y x}=0 \\
\text { (2) }-\frac{\partial \varepsilon_{x}}{\partial y}+\frac{\partial \gamma_{y x}}{\partial x}-\frac{\partial^{2} z}{\partial x \partial y} \gamma_{x N}-\frac{\partial^{2} z}{\partial x^{2}} \gamma_{y N}+\frac{\partial z}{\partial y} \kappa_{x}-\frac{\partial z}{\partial x} \kappa_{x y}=0 \\
\text { (3) } \frac{\partial \gamma_{x N}}{\partial y}-\frac{\partial \gamma_{y N}}{\partial x}+\kappa_{x y}-\kappa_{y x}=0 \\
\text { (4) } \frac{\partial \kappa_{y}}{\partial x}=\frac{\partial \kappa_{x y}}{\partial y} \\
\text { (5) } \frac{\partial \kappa_{x}}{\partial y}=\frac{\partial \kappa_{y x}}{\partial x}
\end{gathered}
$$

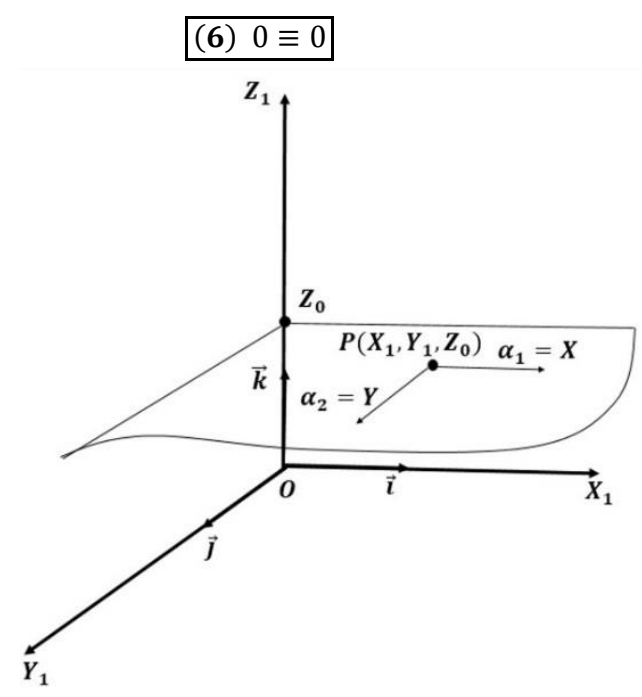

Figure 2. Cartesian plane as reference surface

\subsection{Bi-dimensional elasticity problems in rectangular coordinate. Saint-Venant's equation in plane state}

By substituting the geometric characteristics of the Cartesian plane, Eq. (27), and making $f=$ 0 simultaneously in the new general compatibility equations, Eq. (22), is obtain the compatibility equations of the deformations for the plane state, Eq. (29). Likewise, the geometric equations for this case are obtained, Eq. (30). 


$$
\begin{gathered}
\text { (1) }-\frac{\partial \varepsilon_{y}}{\partial x}+\frac{\partial \gamma_{x y}}{\partial y}=0 \\
\text { (2) }-\frac{\partial \varepsilon_{x}}{\partial y}+\frac{\partial \gamma_{y x}}{\partial x}=0 \\
\varepsilon_{x}=\frac{\partial U_{x}}{\partial x}, \quad \varepsilon_{y}=\frac{\partial U_{y}}{\partial y}, \quad \gamma_{x y}=\frac{\partial U_{y}}{\partial x}, \quad \gamma_{y x}=\frac{\partial U_{x}}{\partial y}
\end{gathered}
$$

By defining $\omega_{x y}=\gamma_{x y}+\gamma_{y x}$ like in the bi-dimensional elasticity theory in Cartesian coordinate, the geometric equations, Eq. (30), become in Eq. (31), and therefore, the typical Saint-Venant's equation in Cartesian coordinate for the plane state is obtained, Eq. (32) [16, 17].

$$
\begin{gathered}
\varepsilon_{x}=\frac{\partial U_{x}}{\partial x}, \quad \varepsilon_{y}=\frac{\partial U_{y}}{\partial y}, \quad \omega_{x y}=\gamma_{x y}+\gamma_{y x}=\frac{\partial U_{y}}{\partial x}+\frac{\partial U_{x}}{\partial y} \\
\frac{\partial^{2} \omega_{x y}}{\partial x \partial y}=\frac{\partial^{2} \varepsilon_{x}}{\partial y^{2}}+\frac{\partial^{2} \varepsilon_{y}}{\partial x^{2}}
\end{gathered}
$$

\subsection{New compatibility equations of projected deformations on a polar plane}

New compatibility equations on a polar plane, Eq.(34), were obtained, by replacing the geometric characteristics, Eq. (33), (Figure 3) in the general compatibility equations, Eq. (22).

$$
\begin{gathered}
\alpha_{1}=\rho ; \alpha_{2}=\theta ; f=z(\rho, \theta) ; A_{1}=1, A_{2}=\rho, R_{1}=R_{2}=\infty ; \rho_{1}=\infty, \rho_{2}=\rho \\
B_{1}=\frac{\partial f}{\partial \rho} ; B_{2}=\frac{1}{\rho} \frac{\partial f}{\partial \theta} ; \quad C_{11}=-\frac{\partial^{2} f}{\partial \rho^{2}} ; C_{22}=-\frac{1}{\rho} \frac{\partial f}{\partial \rho}-\frac{1}{\rho^{2}} \frac{\partial^{2} f}{\partial \theta^{2}} ; \quad C_{12}=C_{21}=\frac{1}{\rho^{2}} \frac{\partial^{2} f}{\partial \theta^{2}}-\frac{1}{\rho} \frac{\partial^{2} f}{\partial \rho \partial \theta} \\
K_{1}=\left[1+\left(\frac{\partial f}{\partial \rho}\right)^{2}\right]^{1 / 2}=\sec \beta_{1}, K_{2}=\left[1+\left(\frac{1}{\rho} \frac{\partial f}{\partial \theta}\right)^{2}\right]^{1 / 2}=\sec \beta_{2} ; \lambda_{1}=\lambda_{2}=1 \\
\begin{array}{c}
\text { (1) } \frac{\varepsilon_{\rho}}{\rho}-\frac{\varepsilon_{\theta}}{\rho}-\frac{\partial \varepsilon_{\theta}}{\partial \rho}+\frac{1}{\rho} \frac{\partial \gamma_{\rho \theta}}{\partial \theta}+\left(-\frac{1}{\rho} \frac{\partial f}{\partial \rho}-\frac{1}{\rho^{2}} \frac{\partial^{2} f}{\partial \theta^{2}}\right) \gamma_{\rho N}-\left(\frac{1}{\rho^{2}} \frac{\partial f}{\partial \theta}-\frac{1}{\rho} \frac{\partial^{2} f}{\partial \rho \partial \theta}\right) \gamma_{\theta N}+\frac{\partial f}{\partial \rho} \kappa_{\theta}--\frac{1}{\rho} \frac{\partial f}{\partial \theta} \kappa_{\theta} \rho=0 \\
\text { (2) - } \frac{1}{\rho} \frac{\partial \varepsilon_{\rho}}{\partial \theta}+\frac{1}{\rho} \gamma_{\rho \theta}+\frac{1}{\rho} \gamma_{\theta \rho}+\frac{\partial \gamma_{\theta \rho}}{\partial \rho}-\left(\frac{1}{\rho^{2}} \frac{\partial f}{\partial \theta}-\frac{1}{\rho} \frac{\partial^{2} f}{\partial \rho \partial \theta}\right) \gamma_{\rho N}-\frac{\partial^{2} f}{\partial \rho^{2}} \gamma_{\theta N}+\frac{1}{\rho} \frac{\partial f}{\partial \theta} \kappa_{\rho}--\frac{\partial f}{\partial \rho} \kappa_{\rho \theta}=0 \\
\text { (3) } \frac{1}{\rho} \frac{\partial \gamma_{\rho N}}{\partial \theta}-\frac{1}{\rho} \gamma_{\theta N}-\frac{\partial \gamma_{\theta N}}{\partial \rho}+\kappa_{\rho \theta}-\kappa_{\theta \rho}=0 \\
\text { (4) } \frac{1}{\rho} \kappa_{\rho}-\frac{1}{\rho} \kappa_{\theta}-\frac{\partial \kappa_{\theta}}{\partial \rho}+\frac{1}{\rho} \frac{\partial \kappa_{\rho \theta}}{\partial \theta}=0 \\
\text { (5) }-\frac{1}{\rho} \frac{\partial \kappa_{\rho}}{\partial \theta}+\frac{1}{\rho} \kappa_{\rho \theta}+\frac{1}{\rho} \kappa_{\theta \rho}+\frac{\partial \kappa_{\theta \rho}}{\partial \rho}=0 \\
(6) 0 \equiv 0
\end{array}
\end{gathered}
$$




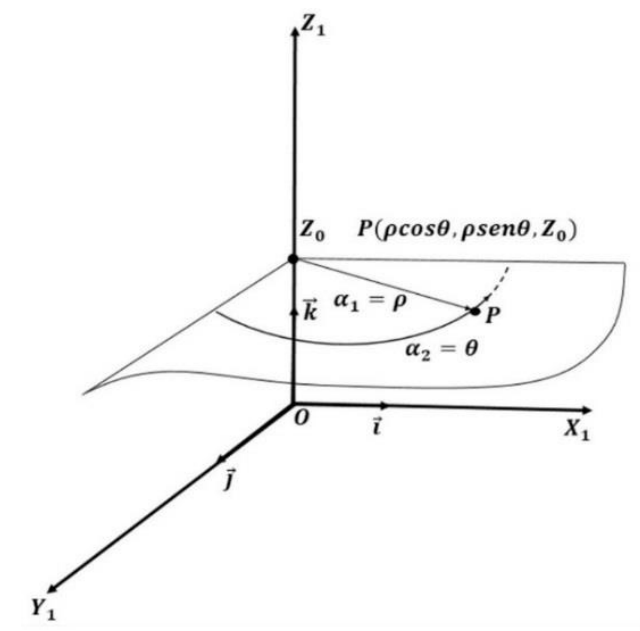

Figure 3. Polar plane as reference surface $[1,2,18]$

3.6. New compatibility equations of the projected deformations on a general cylindrical surface of radius $r_{0}$

The new compatibility equations on a general cylindrical surface of radius $r_{0}$ are obtained, Eq. (36), by substituting the geometric characteristics, Eq. (35), (Figure 4) in the general compatibility equations, Eq. (22). The practical applications of the new compatibility equations, Eq. (36), relies on the calculus of the rotational bodies with Non-axial-symmetric character of directresses: straight (coaxial cylinder), breaking (Güira tank) (Figure 5), hyperbolical (Cooling Towers), among others [1921].

$$
\begin{aligned}
& \alpha_{1}=t, \alpha_{2}=z, f=z(t, z), A_{1}=r_{0}, A_{2}=1, R_{1}=r_{0}, R_{2}=\infty, \rho_{1}=\rho_{2}=\infty \\
& B_{1}=\operatorname{Tan} \beta_{1}=\frac{\frac{\partial f(t, z)}{\partial t}}{r_{0}+f(t, z)}, \quad B_{2}=\operatorname{Tan} \beta_{2}=\frac{\partial f(t, z)}{\partial z} \\
& K_{1}=\left[\left(1+\frac{f(t, z)}{r_{0}}\right)^{2}+\left(\frac{1}{r_{0}} \frac{\partial f(t, z)}{\partial t}\right)^{2}\right]^{1 / 2} ; \quad K_{2}=\left[1+\left(\frac{\partial f(t, z)}{\partial z}\right)^{2}\right]^{1 / 2} \\
& \text { (1) }-\frac{1}{r_{0}} \frac{\partial \varepsilon_{z}}{\partial t}+\frac{\partial \gamma_{t z}}{\partial z}+\frac{1}{r_{0}} \frac{\partial f}{\partial z} \gamma_{z t}-\frac{\partial^{2} f}{\partial z^{2}} \gamma_{t N}-\left(\frac{1}{r_{0}{ }^{2}+r_{0} f} \frac{\partial f}{\partial t} \frac{\partial f}{\partial z}-\frac{1}{r_{0}} \frac{\partial^{2} f}{\partial t \partial z}\right) \gamma_{z N}+\frac{1}{r_{0}+f} \frac{\partial f}{\partial t}\left(1+\frac{f}{r_{0}}\right) \kappa_{z}-\frac{\partial f}{\partial z} \kappa_{z t}- \\
& \left(1+\frac{f}{r_{0}}\right) \tau_{z}=0 \\
& \text { (2) }-\frac{\partial \varepsilon_{t}}{\partial z}-\frac{1}{r_{0}^{2}+r_{0} f} \frac{\partial f}{\partial t} \gamma_{z t}+\frac{1}{r_{0}} \frac{\partial \gamma_{z t}}{\partial t}-\frac{\partial}{\partial z}\left(\frac{1}{r_{0}+f} \frac{\partial f}{\partial t}\right) \gamma_{t N}+\left[\frac{1}{r_{0}}+\frac{1}{r_{0}}\left(\frac{1}{r_{0}+f} \frac{\partial f}{\partial t}\right)^{2}-\frac{1}{r_{0}} \frac{\partial}{\partial t}\left(\frac{1}{r_{0}+f} \frac{\partial f}{\partial t}\right)\right] \gamma_{z N} \frac{\partial f}{\partial z} \kappa_{t}- \\
& \left(1+\frac{f}{r_{0}}\right) \frac{1}{r_{0}+f} \frac{\partial f}{\partial t} \kappa_{t z}+\tau_{t}=0 \\
& \text { (3) } \frac{\gamma_{z t}}{r_{0}}+\frac{\partial \gamma_{t N}}{\partial z}-\frac{1}{r_{0}{ }^{2}+r_{0} f} \frac{\partial f}{\partial t} \gamma_{z N}-\frac{\partial \gamma_{z N}}{\partial t}+\kappa_{t z}-\left(1+\frac{f}{r_{0}}\right) \kappa_{z t}=0 \\
& \text { (4) }-\frac{1}{r_{0}} \frac{\partial \kappa_{z}}{\partial t}+\frac{\partial \kappa_{t z}}{\partial z}-\frac{1}{r_{0}} \tau_{z}=0 \\
& \text { (5) }-\frac{\partial \kappa_{t}}{\partial z}+\frac{1}{r_{0}} \frac{\partial \kappa_{z t}}{\partial t}=0 \\
& \text { (6) }-\frac{1}{r_{0}} \kappa_{z}-\frac{\partial \tau_{t}}{\partial z}+\frac{1}{r_{0}} \frac{\partial \tau_{z}}{\partial t}=0
\end{aligned}
$$




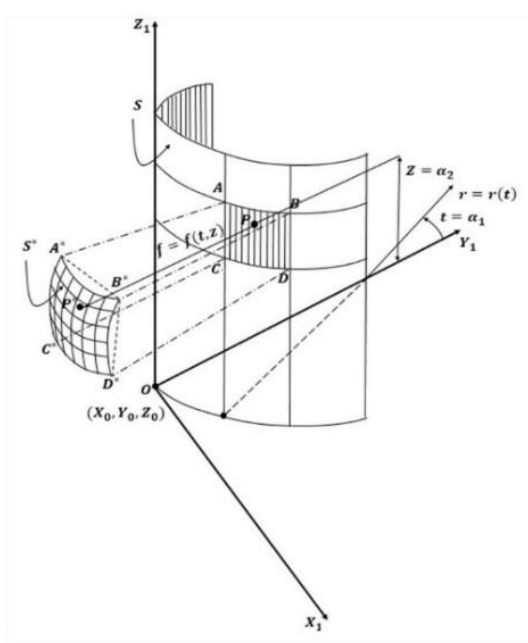

Figure 4. General cylindrical reference surface [1, 2]

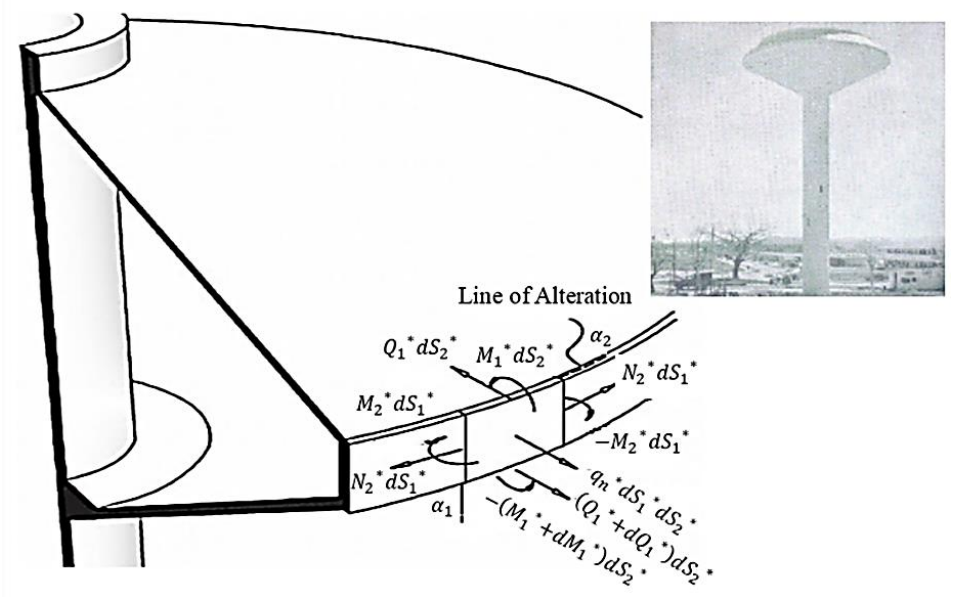

Figure 5. General cylindrical reference surface [1, 2]

3.7. New compatibility equations of the projected deformations on a coaxial cylinder of constant radius $R$

The compatibility equations of projected shell deformations on a coaxial cylinder, Eq. (38), were obtained by substituting the geometric characteristics of the coaxial cylinder, Eq. (37), in the general compatibility equations, Eq. (22). In Eq. (37), the parameter $\theta$ is measured in a positive trigonometric direction and the relative function $f=f\left(\alpha_{1}, \alpha_{2}\right)$ only depends on the independent variable $z$. The practical application of the deformations compatibility equations, Eq. (38), lies in the calculus of the rotational bodies with axial-symmetric character [11-15].

$$
\begin{gathered}
\alpha_{1}=\theta, \alpha_{2}=z, f=f(z), A_{1}=R, A_{2}=1, R_{1}=R, R_{2}=\infty, \rho_{1}=\rho_{2}=\infty, B_{1}=\operatorname{Tan} \beta_{1}=0 \\
B_{2}=\operatorname{Tan} \beta_{2}=\frac{d f(z)}{d z}, K_{1}=1+\frac{f}{R}, K_{2}=\left[1+\left(\frac{d f(z)}{d z}\right)^{2}\right]^{1 / 2}, \lambda_{1}=K_{1}, \cos \beta_{1}=1, \\
\lambda_{2}=1, \cos \beta_{2}=\frac{1}{K_{2}}, C_{11}=\frac{1}{R}, C_{22}=-\frac{d^{2} f}{d z^{2}}, C_{12}=C_{21}=0 \\
\text { (1) }-R \frac{d f}{d z} \frac{d \varepsilon_{\theta}}{d z}+R \kappa_{\theta}+\frac{d f}{d z} \gamma_{Z N}=0 \\
\text { (2) } \frac{\gamma_{Z \theta}}{R}-K_{1} \kappa_{\theta Z}+\frac{d \gamma_{\theta N}}{d z}=0 \\
\text { (3) } \frac{1}{R}\left(\frac{d f}{d z}\right)^{2} \kappa_{Z}-\frac{d^{2} f}{d z^{2}} \kappa_{\theta}+\frac{d f}{d z} \frac{d \kappa_{\theta}}{d z}=0 \\
\text { (4) } \gamma_{\theta Z}-\frac{d f}{d z} \gamma_{\theta N}=0
\end{gathered}
$$




\section{Conclusions}

The new deformations compatibility equations, Eq. (22), using the mathematical assistant "Wolfram Mathematic 9" can be programmed in the construction of the inverse formulation (type Airy) for the mathematical modeling of different shell problems [19, 20, 22-26]. The integration of the non-linear geometrical expressed in the relative coordinate system with projected deformations may be included in the inverse formulation.

The deformations compatibility equations can be obtained at a time, either first or second type Saint-Venant's relationships through the method proposed (appendix case C) $[9,16,17,27]$.

The proposal of the operator polynomial $P_{i}^{N}=P_{i}^{N}\left(X_{1}, X_{2}, X_{3}, \ldots X_{\mathrm{m}}\right), \forall i, m \in \mathbb{N}$ provides deformations compatibility equations of different orders. The method can be applied to cases of geometric equations involving on lineal terms.

The deformations compatibility equations of Reissner-Mindlin, Eq. (23), and Love-Kirchhoff flexion theory, Eq. (25), constitute particular cases of the new general compatibility equations, Eq. (22).

The general equations, Eq. (22), provide the possibility to obtain new compatibility equations when selecting the reference surface $(S)$. They also offer the advantage of choosing the reference surface $(S)$ that generates the least analytical complexity for each problem, so that complex geometric shells can be calculated.

The compatibility conditions of the deformations established by A.L. Goldenveizer, Eq. (5), are only valid when the middle surface of the shell is expressed in Gauss's curvilinear orthogonal coordinate system $(\omega=\pi / 2)$.

The new method allowed to obtain the compatibility equations with projected deformations into the relative coordinate which includes the orthogonal coordinate system $(\omega=\pi / 2)$ (Figure 4).

The principle of the indeterminate coefficient made it possible the formation of the new method since the geometrical equations constitute elements of a Euclidian's vectorial space $(E)$.

Author Contributions: All authors contributed to the study conception, design, and analysis. All authors have read and agreed to the published version of the manuscript.

Funding: This research received no external funding.

Acknowledgments: The authors would like to express their gratitude to Ph.D. Ángel Emilio Castañeda Hevia for his support. The authors wish to thank the organizations that supported the research: The Central University of Las Villas (UCLV).

Conflicts of Interest: The authors declare no conflict of interest.

\section{Appendix A. Bi-dimensional problem in Cartesian coordinates}

In this case, the geometric equations (A.1) are made of differential operators that directly operate on the displacements $(U$ and $V$ ).

1. Geometric equations in Cartesian coordinates for the plane state

(1) $\varepsilon_{X}=\frac{\partial U}{\partial X} ;(2) \varepsilon_{Y}=\frac{\partial V}{\partial Y} ;$ (3) $\gamma_{X Y}=\frac{\partial U}{\partial Y}+\frac{\partial V}{\partial X}$

$\vec{\delta}(x, y)=U(x, y) \vec{I}+V(x, y) \vec{\jmath} \rightarrow$ Displacement field

2. Proposition of the operator polynomial

2.1. Starting with a first-degree $\left(P_{i}^{(1)}\right)$ (A.2) operator polynomial and following the steps already described, the solution obtained is trivial (A.3), which implies the non-existence of first-degree deformations compatibility equations for this problem.

$$
\begin{aligned}
& P_{i}^{(1)}=P^{(1)}(X, Y)_{i}=A_{i} \varepsilon_{i}+B_{i} \frac{\partial \varepsilon_{i}}{\partial X}+C_{i} \frac{\partial \varepsilon_{i}}{\partial Y}, \forall i \in \mathbb{N} \\
& A_{1}=A_{2}=A_{3}=B_{1}=B_{2}=B_{3}=C_{1}=C_{2}=C_{3}=0
\end{aligned}
$$


2.2. Proposition of the second-degree operator polynomial $\left(P^{(2)}{ }_{i}\right)(\mathrm{A} .4)$

$$
P_{i}^{(2)}=P^{(2)}(X, Y)_{i}=A_{i} \varepsilon_{i}+B_{i} \frac{\partial \varepsilon_{i}}{\partial X}+C_{i} \frac{\partial \varepsilon_{i}}{\partial Y}+D_{i} \frac{\partial^{2} \varepsilon_{i}}{\partial X^{2}}+E_{i} \frac{\partial^{2} \varepsilon_{i}}{\partial Y^{2}}++F_{i} \frac{\partial^{2} \varepsilon_{i}}{\partial X \partial Y} \forall i \in \mathbb{N}
$$

3. Derivation of each geometric equation (A.1) according to the analytical structure of the operator polynomial proposed in the previous step $\left(P^{(2)}{ }_{i}\right)$ until obtaining all the $\left(P^{(2)}{ }_{i}\right)$, being $i$ the quantity of the problem geometric equations. Successive derivations of the first geometric equation of (A.1), (A.5).

$$
\left.\left.\left.\left.\varepsilon_{X}=\frac{\partial U}{\partial X} \Rightarrow a\right) \frac{\partial \varepsilon_{X}}{\partial X}=\frac{\partial^{2} U}{\partial X^{2}}, b\right) \frac{\partial \varepsilon_{X}}{\partial Y}=\frac{\partial^{2} U}{\partial Y \partial X} \text { c) } \frac{\partial^{2} \varepsilon_{X}}{\partial X^{2}}=\frac{\partial U^{3}}{\partial X^{3}} ; d\right) \frac{\partial^{2} \varepsilon_{X}}{\partial Y^{2}}=\frac{\partial U^{3}}{\partial Y^{2} \partial X}, e\right) \frac{\partial^{2} \varepsilon_{X}}{\partial X \partial Y}=\frac{\partial U^{3}}{\partial X^{2} \partial Y}
$$

Obtaining the first operator polynomial $P^{(2)}{ }_{1}\left(\varepsilon_{X}\right)$ (A.6):

$$
P_{1}^{(2)}\left(\varepsilon_{X}\right)=A_{1} \frac{\partial U}{\partial X}+B_{1} \frac{\partial^{2} U}{\partial X^{2}}+C_{1} \frac{\partial^{2} U}{\partial Y \partial X}+D_{1} \frac{\partial U^{3}}{\partial X^{3}}+E_{1} \frac{\partial U^{3}}{\partial Y^{2} \partial X}+F_{1} \frac{\partial U^{3}}{\partial X^{2} \partial Y}
$$

The rest of the operator polynomials $\left(P^{(2)}{ }_{2}\left(\varepsilon_{y}\right)\right.$ y $\left.P^{(2)}{ }_{3}\left(\gamma_{X Y}\right)\right)$ is obtained for the second and third geometric equations respectively (A.7) by doing the successive derivation with respect to the second and third geometric equations (A.1).

$$
\begin{gathered}
P^{(2)}{ }_{2}\left(\varepsilon_{y}\right)=A_{2} \frac{\partial V}{\partial Y}+B_{2} \frac{\partial^{2} V}{\partial X \partial Y}+C_{2} \frac{\partial^{2} V}{\partial Y^{2}}+D_{2} \frac{\partial V^{3}}{\partial X^{2} \partial Y}+E_{2} \frac{\partial V^{3}}{\partial Y^{3}}+F_{2} \frac{\partial V^{3}}{\partial X \partial Y^{2}} \\
P^{(2)}{ }_{3}\left(\gamma_{X Y}\right)=A_{3}\left(\frac{\partial U}{\partial Y}+\frac{\partial V}{\partial X}\right)+B_{3}\left(\frac{\partial^{2} U}{\partial X \partial Y}+\frac{\partial^{2} V}{\partial X^{2}}\right)+C_{3}\left(\frac{\partial^{2} U}{\partial Y^{2}}+\frac{\partial^{2} V}{\partial Y \partial X}\right)+E_{3}\left(\frac{\partial U^{3}}{\partial Y^{3}}+\frac{\partial \mathrm{V}^{3}}{\partial Y^{2} \partial \mathrm{X}}\right)+\mathrm{F}_{3}\left(\frac{\partial \mathrm{U}^{3}}{\partial \mathrm{X} \partial \mathrm{Y}^{2}}+\frac{\partial \mathrm{V}^{3}}{\partial \mathrm{X}^{2} \partial \mathrm{Y}}\right)
\end{gathered}
$$

Formation of the lineal combination between the operator polynomials (A.6 and A.7) until the fundamental identity is formed (A.8): $\sum_{i=1}^{n} P^{(2)}{ }_{i}=0 \Rightarrow P^{(2)}{ }_{1}\left(\varepsilon_{X}\right)+P^{(2)}{ }_{2}\left(\varepsilon_{Y}\right)+P^{(2)}{ }_{3}\left(\gamma_{X Y}\right)=0$

$$
\begin{gathered}
A_{1} \frac{\partial U}{\partial X}+B_{1} \frac{\partial^{2} U}{\partial X^{2}}+C_{1} \frac{\partial^{2} U}{\partial Y \partial X}+D_{1} \frac{\partial U^{3}}{\partial X^{3}}+E_{1} \frac{\partial U^{3}}{\partial Y^{2} \partial X}+F_{1} \frac{\partial U^{3}}{\partial X^{2} \partial Y}+A_{2} \frac{\partial V}{\partial Y}+B_{2} \frac{\partial^{2} V}{\partial X \partial Y}+C_{2} \frac{\partial^{2} V}{\partial Y^{2}}+D_{2} \frac{\partial V^{3}}{\partial X^{2} \partial Y}+E_{2} \frac{\partial V^{3}}{\partial Y^{3}}+ \\
F_{2} \frac{\partial V^{3}}{\partial X \partial Y^{2}}+A_{3}\left(\frac{\partial U}{\partial Y}+\frac{\partial V}{\partial X}\right)+B_{3}\left(\frac{\partial^{2} U}{\partial X \partial Y}+\frac{\partial^{2} V}{\partial X^{2}}\right)+C_{3}\left(\frac{\partial^{2} U}{\partial Y^{2}}+\frac{\partial^{2} V}{\partial Y \partial X}\right)+D_{3}\left(\frac{\partial U^{3}}{\partial X^{2} \partial Y}+\frac{\partial V^{3}}{\partial X^{3}}\right)+E_{3}\left(\frac{\partial U^{3}}{\partial Y^{3}}+\frac{\partial V^{3}}{\partial Y^{2} \partial X}\right)+ \\
F_{3}\left(\frac{\partial U^{3}}{\partial X \partial Y^{2}}+\frac{\partial V^{3}}{\partial X^{2} \partial Y}\right)=0
\end{gathered}
$$

4. Application of the indeterminate coefficient method (ICM)

4.1. Obtaining of the lineal homogenous equation system that provides the formation of the fundamental identity (A.8) in the previous step. The solution of the fundamental identity (A.8), offered a lineal homogenous equation system of 3 equations with 2 unknown factors (A.9).

$$
\begin{aligned}
& E_{1}+F_{3}=0 \\
& D_{2}+F_{3}=0
\end{aligned}
$$

Resolution of the lineal homogenous equation system (1.10)

$$
\left.\begin{array}{c}
E_{1}+F_{3}=0 \Rightarrow E_{1}=-F_{3} \\
D_{2}+F_{3}=0 \Rightarrow D_{2}=-F_{3}
\end{array}\right\} E_{1}=D_{2}=-F_{3} \Rightarrow S:\left\{\left(E_{1}, D_{2}, F_{3}\right) \in \mathbb{R}: E_{1}=D_{2}=-F_{3}\right\}
$$

5. Interpretation of the solution of the lineal homogenous equation system. The solution of the lineal homogenous equation system (A.10) provided only one free variable, which implies the existence of one deformations compatibility equation. By recalling the analytical structure of the second-degree operator polynomial $\left(P^{(2)}{ }_{i}\right)(\mathrm{A} .4)$, the Saint-Venant's equation for the bi-dimensional elasticity theory in Cartesian coordinates is obtained (A.11) (A.15).

$$
E_{1}=D_{2}=-F_{3} \Rightarrow \frac{\partial^{2} \varepsilon_{X}}{\partial Y^{2}}+\frac{\partial^{2} \varepsilon_{Y}}{\partial X^{2}}-\frac{\partial^{2} \gamma_{X Y}}{\partial X \partial Y}=0
$$

If a third-degree $\left(P^{(3)}\right)$ operator polynomial (A.12) were used instead of a seconddegree $\left(P^{(2)}{ }_{i}\right)$ (A.4) polynomial the solution (A.13) would be obtained. 


$$
\begin{gathered}
P_{i}^{(3)}=P^{(3)}(X, Y)_{i}=A_{i} \varepsilon_{i}+B_{i} \frac{\partial \varepsilon_{i}}{\partial X}+C_{i} \frac{\partial \varepsilon_{i}}{\partial Y}+D_{i} \frac{\partial^{2} \varepsilon_{i}}{\partial X^{2}}+E_{i} \frac{\partial^{2} \varepsilon_{i}}{\partial Y^{2}}+F_{i} \frac{\partial^{2} \varepsilon_{i}}{\partial X \partial Y}+G_{i} \frac{\partial^{3} \varepsilon_{i}}{\partial X^{3}}+H_{i} \frac{\partial^{3} \varepsilon_{i}}{\partial Y^{3}}+I_{i} \frac{\partial^{3} \varepsilon_{i}}{\partial X^{2} \partial Y}+ \\
J_{i} \frac{\partial^{3} \varepsilon_{i}}{\partial X \partial Y^{2}} \\
S:\left\{\left(E_{1}, D_{2}, F_{3}, H_{1}, I_{2} y J_{3}\right) \in \mathbb{R}: E_{1}=D_{2}=-F_{3} ; H_{1}=I_{2}=-J_{3}\right\}
\end{gathered}
$$

The solution (A.13) expresses the existence of two deformations compatibility equations (A.14) which contain the Saint-Venant's equation (A.11), due to the fact of using a thirddegree $\left(P^{(3)}{ }_{i}\right)$ operator polynomial (A.12) that has a second-degree $\left(P^{(2)}{ }_{i}\right)$ operator polynomial (A.2) in its analytical structure that was used for obtaining the Saint-Venant's equation (A.11). It can also be observed in the solution (A.14) that the identity obtained from $\left(H_{1}=I_{2}=-J_{3}\right)$ is not a new compatibility equation since it is the same Saint-Venant's derivative equation (A.11) with respect to the independent variable $Y$.

$$
\begin{gathered}
E_{1}=D_{2}=-F_{3} \Rightarrow \underbrace{\frac{\partial^{2} \varepsilon_{X}}{\partial Y^{2}}+\frac{\partial^{2} \varepsilon_{X}}{\partial X^{2}}-\frac{\partial^{2} \gamma_{X Y}}{\partial X \partial Y}=0}_{\text {Saint-Venant }} \\
H_{1}=I_{2}=-J_{3} \Rightarrow \frac{\partial}{\partial Y}(\underbrace{\frac{\partial^{2} \varepsilon_{X}}{\partial Y^{2}}+\frac{\partial^{2} \varepsilon_{X}}{\partial X^{2}}-\frac{\partial^{2} \gamma_{X Y}}{\partial X \partial Y}}_{\text {Saint-Venant }})=0
\end{gathered}
$$

The solution (A.14) expresses that the geometric equations of the bi-dimensional problem in Cartesian coordinates do not require deformations compatibility equations higher than the second order. The expression (A.15) shows a summary of the results of the operator polynomial of different orders, and the convergence to the Saint-Venant's solution (A.11) obtained with an operator polynomial of second degree $\left(P^{(2)}{ }_{i}\right)$.

$P^{(1)}(X, Y)_{i} \Rightarrow$ Null solution

$$
\begin{gathered}
P^{(2)}(X, Y)_{i} \Rightarrow \frac{\partial^{2} \varepsilon_{X}}{\partial Y^{2}}+\frac{\partial^{2} \varepsilon_{X}}{\partial X^{2}}-\frac{\partial^{2} \gamma_{X Y}}{\partial X \partial Y}=0 \text { (problem solution) } \\
P^{(3)}(X, Y)_{i} \Rightarrow\left\{\begin{array}{c}
\frac{\partial^{2} \varepsilon_{X}}{\partial Y^{2}}+\frac{\partial^{2} \varepsilon_{X}}{\partial X^{2}}-\frac{\partial^{2} \gamma_{X Y}}{\partial X \partial Y}=0 \\
\frac{\partial}{\partial Y}\left(\frac{\partial^{2} \varepsilon_{X}}{\partial Y^{2}}+\frac{\partial^{2} \varepsilon_{X}}{\partial X^{2}}-\frac{\partial^{2} \gamma_{X Y}}{\partial X \partial Y}\right)=0
\end{array}\right.
\end{gathered}
$$

\section{Appendix B. Bi-dimensional problems in Polar coordinates}

In this case, the geometric equations (B.16) are made up of functions that multiply the differential operators, which function directly on the displacements $\left(U_{\rho}\right.$ and $\left.U_{\theta}\right)$, and of displacements that are not operated by differential operators.

By applying the proposed procedure to the geometric equations (B.16) using the second-degree operator polynomial $\left(P^{(2)}(\rho, \theta)_{i}\right)$ (B.17) in polar coordinates the solution set (B.18) is obtained. The solution set (B.18) offered only one free variable; which represents the existence of one deformations compatibility equation. In addition, by means of the analytical structure of the operator polynomial of second degree $\left(P^{(2)}(\rho, \theta)_{i}\right)$ (B.17), the Saint-Venant's equation for the bi-dimensional elasticity theory in Polar coordinates is obtained (B.19).

Geometric equations in Polar coordinates (B.16)

$$
\text { (1) } \varepsilon_{\rho}=\frac{\partial U_{\rho}}{\partial \rho}, \text { (2) } \varepsilon_{\theta}=\frac{U_{\rho}}{\rho}+\frac{1}{\rho} \frac{\partial U_{\theta}}{\partial \theta} \text {, (3) } \gamma_{\rho \theta}=\frac{1}{\rho} \frac{\partial U_{\rho}}{\partial \theta}-\frac{U_{\theta}}{\rho}+\frac{\partial U_{\theta}}{\partial \rho}
$$

Proposition of the second-degree operator polynomial type $P^{(2)}(\rho, \theta)_{i}$ in Polar coordinates (B.17).

$$
P^{(2)}(\rho, \theta)_{i}=A_{i} \varepsilon_{i}+B_{i} \frac{\partial \varepsilon_{i}}{\partial \rho}+C_{i} \frac{\partial \varepsilon_{i}}{\partial \theta}+D_{i} \frac{\partial^{2} \varepsilon_{i}}{\partial \rho^{2}}+E_{i} \frac{\partial^{2} \varepsilon_{i}}{\partial \theta^{2}}+F_{i} \frac{\partial^{2} \varepsilon_{i}}{\partial \rho \partial \theta}
$$

Solution set for the proposal $P^{(2)}(\rho, \theta)_{i}$ (B.18) 


$$
\begin{gathered}
S:\left\{B_{1}, F_{3}, B_{2}, C_{3}, D_{2}, E_{1}\right\}, \in \mathbb{R}: \\
\left\{-B_{1}=\frac{E_{1}}{\rho}=2 B_{2}=\rho D_{2}=-\frac{C_{3}}{\rho}=-F_{3}\right\}
\end{gathered}
$$

Compatibility equation of the deformations in Polar coordinates (B.19)

$$
-\frac{\partial \varepsilon_{\rho}}{\partial \rho}+\frac{1}{\rho} \frac{\partial^{2} \varepsilon_{\rho}}{\partial \theta^{2}}+2 \frac{\partial \varepsilon_{\theta}}{\partial \rho}+\rho \frac{\partial^{2} \varepsilon_{\theta}}{\partial \rho^{2}}-\frac{1}{\rho} \frac{\partial \gamma_{\rho \theta}}{\partial \theta}-\frac{\partial^{2} \gamma_{\rho \theta}}{\partial \rho \partial \theta}=0
$$

Similarly to case $A$ (bi-dimensional problems in Cartesian coordinates), if a first-degree operator polynomial $\left[P^{(1)}(\rho, \theta)_{i}\right]$ had been used in Polar coordinates, then the solution obtained would have been trivial, which implies the nonexistence of first-degree compatibility equations for this problem. Equally, if an operator polynomial higher than second degree were used, the solution of the lineal combination of the partial derivative equation (B.19) would be obtained.

\section{Appendix C. Bi-dimensional problems in Polar coordinates}

In the previous cases ( $\mathrm{A}$ and $\mathrm{B})$, operators polynomial were proposed in two independent variables (A.4, A.12 and B.17), either for Cartesian coordinate problems (case A) or Polar ones (case B). The application of the proposed procedure to the tridimensional elasticity theory geometric equations (C.20) for an operator polynomial in three independent variables $(X, Y, Z)$ (C.21) is shown below.

1. Geometric equations in Cartesian coordinates

(1) $\varepsilon_{X}=\frac{\partial U}{\partial X}$, (2) $\varepsilon_{Y}=\frac{\partial V}{\partial Y}$, (3) $\varepsilon_{Z}=\frac{\partial W}{\partial Z}$, (4) $\gamma_{X Y}=\frac{\partial U}{\partial Y}+\frac{\partial V}{\partial X}$ (5) $\gamma_{X Z}=\frac{\partial U}{\partial Z}+\frac{\partial W}{\partial X}$, (6) $\gamma_{Y Z}=\frac{\partial V}{\partial Z}+$

$$
\frac{\partial W}{\partial Y}
$$

Proposal of the second-degree polynomial type $P^{(2)}(X, Y, Z)$ (C.21)

$$
P^{(2)}(X, Y, Z)_{i}=A_{i} \varepsilon_{i}+B_{i} \frac{\partial \varepsilon_{i}}{\partial X}+C_{i} \frac{\partial \varepsilon_{i}}{\partial Y}+D_{i} \frac{\partial \varepsilon_{i}}{\partial Z}+E_{i} \frac{\partial^{2} \varepsilon_{i}}{\partial X^{2}}+F_{i} \frac{\partial^{2} \varepsilon_{i}}{\partial Y^{2}}+G_{i} \frac{\partial^{2} \varepsilon_{i}}{\partial Z^{2}}+H_{i} \frac{\partial^{2} \varepsilon_{i}}{\partial X \partial Y}+I_{i} \frac{\partial^{2} \varepsilon_{i}}{\partial X \partial Z}+J_{i} \frac{\partial^{2} \varepsilon_{i}}{\partial Y \partial Z}
$$

2. Formation of the lineal combination between the six operator polynomials (C.22)

$$
\begin{gathered}
\sum_{i=1}^{n} P^{(2)}(X, Y, Z)_{i}=0 \Rightarrow P^{(2)}{ }_{1}\left(\varepsilon_{X}\right)+P^{(2)}{ }_{2}\left(\varepsilon_{Y}\right)+P^{(2)}{ }_{3}\left(\varepsilon_{Z}\right)+P^{(2)}{ }_{4}\left(\gamma_{X Y}\right)+P^{(2)}{ }_{5}\left(\gamma_{X Z}\right)+ \\
P^{(2)}{ }_{6}\left(\gamma_{Y Z}\right)=0
\end{gathered}
$$

3. Application of the Indeterminate coefficient method (ICM)

The resolution of the fundamental identity (C.22) offered a lineal homogenous equation system of 18 equations with 24 unknown factors, whose solution is shown in the expression (A.12). The solution (C.23) offered six free variables, which imply the existence of six deformations compatibility equations.

$$
\begin{gathered}
S:\left\{H_{4}, G_{4} I_{5}, F_{5}, E_{6}, J_{6}\right\} \in \mathbb{R}:\left\{\left(F_{1}=E_{2}=-H_{4}\right) ;\left(E_{3}=G_{1}=-I_{5}\right) ;\left(G_{2}=F_{3}=-J_{6}\right)\right\} \\
\left\{\left(H_{6}=J_{4}=-F_{5} \cup I_{2}=2 F_{5}\right) ;\left(J_{5}=I_{6}=-G_{4} \cup H_{3}=2 G_{4}\right) ; I_{4}=H_{5}=-E_{6} \cup J_{1}=2 E_{6}\right\}
\end{gathered}
$$

The six Saint-Venant's equations for the tridimensional elasticity theory in Cartesian coordinates are obtained at a time (C.24 and C.25).

First- type Ratios (C.24)

$$
\begin{aligned}
& \left(F_{1}=E_{2}=-H_{4}\right) \Rightarrow \frac{\partial^{2} \gamma_{X Y}}{\partial X \partial Y}=\frac{\partial^{2} \varepsilon_{X}}{\partial Y^{2}}+\frac{\partial^{2} \varepsilon_{Y}}{\partial X^{2}} \\
& \left(E_{3}=G_{1}=-I_{5}\right) \Rightarrow \frac{\partial^{2} \gamma_{X Z}}{\partial X \partial Z}=\frac{\partial^{2} \varepsilon_{X}}{\partial Z^{2}}+\frac{\partial^{2} \varepsilon_{Z}}{\partial X^{2}} \\
& \left(G_{2}=F_{3}=-J_{6}\right) \Rightarrow \frac{\partial^{2} \gamma_{Y Z}}{\partial Y \partial Z}=\frac{\partial^{2} \varepsilon_{Y}}{\partial Z^{2}}+\frac{\partial^{2} \varepsilon_{Z}}{\partial Y^{2}}
\end{aligned}
$$

Second type Ratios (C.25)

$$
\begin{aligned}
& \begin{array}{c}
\left(H_{6}=J_{4}=-F_{5}\right. \\
\left.\quad \cup I_{2}=2 F_{5}\right)
\end{array} \Rightarrow \frac{\partial^{2} \varepsilon_{Y}}{\partial X \partial Z}=\frac{1}{2} \frac{\partial}{\partial Y}\left(\frac{\partial \gamma_{X Y}}{\partial Z}-\frac{\partial \gamma_{X Z}}{\partial Y}+\frac{\partial \gamma_{Y Z}}{\partial X}\right) \\
& \begin{array}{c}
\left(J_{5}=I_{6}=-G_{4}\right. \\
\left.\cup H_{3}=2 G_{4}\right)
\end{array} \Rightarrow \frac{\partial^{2} \varepsilon_{Z}}{\partial X \partial Y}=\frac{1}{2} \frac{\partial}{\partial Z}\left(-\frac{\partial \gamma_{X Y}}{\partial Z}+\frac{\partial \gamma_{X Z}}{\partial Y}+\frac{\partial \gamma_{Y Z}}{\partial X}\right)
\end{aligned}
$$




$$
\begin{gathered}
\left(I_{4}=H_{5}=-E_{6}\right. \\
\left.\cup J_{1}=2 E_{6}\right)
\end{gathered} \Rightarrow \frac{\partial^{2} \varepsilon_{X}}{\partial Y \partial Z}=\frac{1}{2} \frac{\partial}{\partial X}
$$

If an operator polynomial of first degree $\left[P^{(1)}(X, Y, Z)_{i}\right]$ had been used, the solution would be trivial. This involves the nonexistence of first-degree deformations compatibility equations for this problem. Likewise, if an operator polynomial higher than second degree were used, the solution obtained would be the lineal combination of the partial derivative equation (C.24 and C.25).

Nomenclature

$S^{*}$ : Real surface (arbitrary geometry)

$S: \quad$ Reference surface (simpler geometry)

\section{Parameters}

$\left(\alpha_{1}, \alpha_{2}\right)$ : Gauss's Curvilinear orthogonal coordinate

$\left(A_{1}, A_{2}\right)$ : Lame's parameters

$\left(d s_{1}, d s_{2}\right)$ : Arc differentials of the reference surface $S$

$\left(R_{1}, R_{2}\right)$ : Radius of Gaussian curvature of the reference surface $S$

$\left(\rho_{1}, \rho_{2}\right)$ : Radius of the geodesic curvature of the reference surface $S$

$\left(d A, d A^{*}\right)$ : Area differentials of reference surface $S$ and middle surface $\left(\boldsymbol{S}^{*}\right)$

$\left(C_{11}, C_{22}, C_{12}, C_{21}\right)$ : Inverses of the principal curvatures radius of the surface $\left(S^{*}\right)$ expressed in relative coordinate

$\varepsilon_{1}, \varepsilon_{2}$ : Longitudinal Projected deformations

$\gamma_{12}, \gamma_{21}$ : Tangential Projected distortions

$\gamma_{1 N}, \gamma_{2 N}$ : Projected distortions by shear

$\varkappa_{1}, \varkappa_{2}$ : Projected distortions by flexion

$\varkappa_{12}, \varkappa_{21}$ : Projected distortions by twisting

$\tau_{1}, \tau_{2}$ : Projected distortions by twisting according to the normal axis $\left(\overrightarrow{e_{N}}\right)$

$\vec{U}:$ Displacement vector

$U_{1}, U_{2}, U_{N}$ : Displacement vector components $\vec{U}$

$\vec{\Omega}:$ Rotation vector

$\psi_{1}, \psi_{2}, \delta$ : Rotation vector components $\vec{\Omega}$

\section{Acronyms}

HD: Hyper statics Degrees

FD: Freedom Degrees

RCM: Relative Coordinate Method

ICM: Indeterminate Coefficient Method

F.S.S: Fundamental Solution System

\section{References}

1. J.E. Hernández (Pimpo), Unified Approach to the membrane Theory of Shells, Ingeniería Civil, XXIII (1972) $1-34$.

2. J.E. Hernández (Pimpo), Unified Approach to the membrane Theory of Shells, Ingeniería Civil, XXI (1970) 22-43.

3. A.E. Castañeda, W. Cobelo, Y. González, J. Álvarez, A look at half a century of shells foundations, methods of calculation and associate research in Cuba, Revista ingeniería de construcción, 26 (2011) 245-268.

4. J. Álvarez, Inverse formulation of shell in relative coordinate with projected deformations, Instituto Superior Politécnico José Antonio Echeverría (CUJAE), La Habana, 2014.

5. A.L. Goldenveizer, Theory of thin shells. Chapter 2, 3, 6, New York, Pergamon Press, 1961.

6. A.L. Goldenveizer, The thin elastic shell theory. Chapers 2, 3, 6, Editorial Continental S: A. México, México, 1963.

7. A.E. Castañeda, Calculus of shell method in relative coordinate with proyected deformations, Moscú, 1982. 
8. T. Apostol, Calculus. Chapter 6, 7, 9, Technology Institute, California, 1961.

9. W. Flügge, Tensor Analysis and Continuum Mechanics. Chapter 1, 2, Springer-Verlag, Berlin/Götingen/Heildeberg, 1972.

10. A.L. Goldenveizer, J.D. Kaplunov, E.V. Nolde, On Timoshenko-Reissner type theories of plates and shells, International Journal of Solids and Structures, 30 (1993) 675-694.

11. S.P. Timoshenko, K. Woinnowsky, Theory of Plates and Shells. Chapter 1, 2, 3, 4, 1953.

12. R. Szilard, Theories and Applications of Plate and Shells Analysis. Chapter 1, 2, 3, Wiley, New York, 2004.

13. U.V. Novozhilov, The theory of thin Shells. Chapter 1, 2, 3, P. Noordhof Ltd. Groningen, The Netherlands., 1959.

14. W. Flügge, Stresses in Shells. Chapter 1, 2, 3, Springer-Verlag, Berlin/Götingen/Heildeberg, 1960.

15. A.C. Ugural, Stress in Plates and Shells. Chapter 1, 2, 3, Mc. Graw-Hill Book company, New York, 1999.

16. Filonenko-Borodich, Elasticity Theory. Chapers 1, 2, 3, 4, 5, Editorial Platina1963.

17. S.P. Timoshenko, N.J. Goodier, Elasticity Theory. Chapers: 1, 2, 3, 4, 5, McGraw-Hill Book Company, New York, 1951.

18. M.A. Martínez, Membranal theory formulation of the shell with the finite elements method in relative cordinates with projected deformations, Central University "Martha Abreu" of Las Villas (UCLV), Villa Clara, 2000.

19. Mircea-Birsan, On the theory of loaded general cylindrical Cosserat elastic shell, International Journal of Solids and Structures, 44 (2007) 7399-7419.

20. A.E. Albanesi, V. Fachinotti, A. Cardona, Inverse finite element method for large-displacemente beams, International Journal for Numerical Methods in Engineering, (2010).

21. G. Murali, C. Vivek, B. Prasanth, Response of Cooling Towers to Wind Loads, ARPN Journal of Engineering and Applied Sciences 7 (2012) 114-120.

22. E. Reissner, On a generalization of some formulas of the theory of "moderately thick" elastic plates, International Journal of Solids and Structures, 23 (1987) 711-717.

23. M. Ciarletta, S. Chirita, The Saint-Venant problem and principle in elasticity, International Journal of Solids and Structures, 36 (1999) 1949-1964

24. M.L. Bucalmen, K.J. Bathe, Higher-order MITC general Shells elements, International Journal for Numerical Methods in Engineering, (2011).

25. V. Fachinotti, A.E. Albanesi, Finite elements method type inverse for shell with big displacement in elasticity regime, Argentina Association of Computational Mechanics, (2012).

26. J.E. Hernández (Pimpo), L. Álvarez, E. Álvarez, Cálculo numérico de estructuras laminares de revolución utilizando el método de líneas y las EDAs, Investigación Operacional, 21 (2000) 10-45.

27. V.I. Sokolnikoff, Mathematic Theory of Elasticity. Chapter 2, Mc. Graw-Hill Book company1956.

28. J. M. Rotter and A. J. Sadowski, "Cylindrical shell bending theory for orthotropic shells under general axisymmetric pressure distributions," Engineering Structures, vol. 42, pp. 258-265, 92012. 10.1016/j.engstruct.2012.04.024 